\title{
Dynamic Processes, Fixed Points, Endpoints, Asymmetric Structures, and Investigations Related to Caristi, Nadler, and Banach in Uniform Spaces
}

\author{
Kazimierz Włodarczyk and Robert Plebaniak \\ Department of Nonlinear Analysis, Faculty of Mathematics and Computer Science, University of Łódź, Banacha 22, \\ 90-238 Łódź, Poland \\ Correspondence should be addressed to Kazimierz Włodarczyk; wlkzxa@math.uni.lodz.pl
}

Received 30 October 2014; Accepted 15 January 2015

Academic Editor: Ademir F. Pazoto

Copyright ( 2015 K. Włodarczyk and R. Plebaniak. This is an open access article distributed under the Creative Commons Attribution License, which permits unrestricted use, distribution, and reproduction in any medium, provided the original work is properly cited.

\begin{abstract}
In uniform spaces $(X, \mathscr{D})$ with symmetric structures determined by the $\mathscr{D}$-families of pseudometrics which define uniformity in these spaces, the new symmetric and asymmetric structures determined by the $\mathscr{J}$-families of generalized pseudodistances on $X$ are constructed; using these structures the set-valued contractions of two kinds of Nadler type are defined and the new and general theorems concerning the existence of fixed points and endpoints for such contractions are proved. Moreover, using these new structures, the single-valued contractions of two kinds of Banach type are defined and the new and general versions of the Banach uniqueness and iterate approximation of fixed point theorem for uniform spaces are established. Contractions defined and studied here are not necessarily continuous. One of the main key ideas in this paper is the application of our fixed point and endpoint version of Caristi type theorem for dissipative set-valued dynamic systems without lower semicontinuous entropies in uniform spaces with structures determined by $\mathscr{f}$-families. Results are new also in locally convex and metric spaces. Examples are provided.
\end{abstract}

\section{Introduction}

The concepts of the symmetric and asymmetric structures became established and investigated in mathematics and in theoretical computer science and are some creative ideas in fixed point theory by which some fascinating results have been achieved. In the proofs of these results, some deep methods based on those symmetric and asymmetric structures do play very important roles. The range of important applications of these results is enormous.

Let $(X, \mathscr{D})$ be a uniform space with uniformity defined by a saturated family $\mathscr{D}=\left\{d_{\alpha}: \alpha \in \mathscr{A}\right\}$ of pseudometrics $d_{\alpha}: X^{2} \rightarrow[0 ; \infty), \alpha \in \mathscr{A}$, uniformly continuous on $X^{2}$ (D्D-family, for short); here $\mathscr{A}$ is a nonempty index set.

It was discovered that the $\mathscr{J}$-families of generalized pseudodistances defined below generalize: metrics $d$, distances of Tataru [1], $w$-distances of Kada et al. [2], $\tau$-distances of Suzuki [3], and $\tau$-functions of Lin and Du [4] in metric spaces $(X, d)$ and also $\mathscr{D}$-families of pseudometrics and distances of Vályi $[5]$ in uniform spaces $(X, \mathscr{D})$.
Definition 1 (see [6]). Let $(X, \mathscr{D})$ be a Hausdorff uniform space.

(a) The family $\mathscr{J}=\left\{J_{\alpha}: \alpha \in \mathscr{A}\right\}$ of maps $J_{\alpha}: X^{2} \rightarrow$ $[0 ; \infty), \alpha \in \mathscr{A}$, is said to be a $\mathscr{J}$-family of generalized pseudodistances on $X$ ( $\mathscr{F}$-family, for short) if the following two conditions hold:

$$
\begin{aligned}
& (\mathscr{J} 1) \forall_{\alpha \in \mathscr{A}} \forall_{x, y, z \in X}\left\{J_{\alpha}(x, z) \leqslant J_{\alpha}(x, y)+J_{\alpha}(y, z)\right\} . \\
& (\mathscr{J} 2) \text { For any sequences }\left(x_{m}: m \in \mathbb{N} \in \mathbb{N}\right) \\
& \text { and }\left(y_{m}: m \in \mathbb{N}\right) \text { in } X \text { such that } \\
& \forall_{\alpha \in \mathscr{A}}\left\{\lim _{n \rightarrow \infty} \sup _{m>n} J_{\alpha}\left(x_{n}, x_{m}\right)=0\right\} \text { and } \\
& \forall_{\alpha \in \mathscr{A}}\left\{\lim _{m \rightarrow \infty} J_{\alpha}\left(x_{m}, y_{m}\right)=0\right\} \text {, the following } \\
& \text { holds } \forall_{\alpha \in \mathscr{A}}\left\{\lim _{m \rightarrow \infty} d_{\alpha}\left(x_{m}, y_{m}\right)=0\right\} .
\end{aligned}
$$

(b) Define

$$
\begin{aligned}
\mathbb{J}_{(X, \mathscr{D})}= & \left\{\mathscr{J}: \mathscr{J}=\left\{J_{\alpha}: X^{2} \longrightarrow[0 ; \infty), \alpha \in \mathscr{A}\right\}\right. \\
& \text { is a } \mathscr{J} \text {-family on } X\} .
\end{aligned}
$$


Definition 2. Let $(X, d)$ be a metric space.

(a) Then $\mathscr{J}=\left\{J: X^{2} \rightarrow[0 ; \infty)\right\}$ is said to be a $\mathscr{J}$-family on $X$ if $J$ is a generalized pseudodistance on $X$; that is, the following two conditions hold:

(J1) $\forall_{x, y, z \in X}\{J(x, z) \leqslant J(x, y)+J(y, z)\}$.

(J2) For any sequences $\left(x_{m}: m \in \mathbb{N}\right)$ and $\left(y_{m}: m \in\right.$ $\mathbb{N})$ in $X$ such that $\lim _{n \rightarrow \infty} \sup _{m>n} J\left(x_{n}, x_{m}\right)=0$ and $\lim _{m \rightarrow \infty} J\left(x_{m}, y_{m}\right)=0$, the following holds $\lim _{m \rightarrow \infty} d\left(x_{m}, y_{m}\right)=0$.

(b) Define $\rrbracket_{(X, d)}=\left\{\mathscr{J}: \mathscr{J}=\left\{J: X^{2} \rightarrow[0 ; \infty)\right\}\right.$ is a $\mathscr{J}$-family on $X\}$.

In the following remark, we list some basic properties of $\mathbb{J}_{(X, \mathscr{D})}$

Remark 3. Let $(X, \mathscr{D})$ be a Hausdorff uniform space.

(a) $\mathscr{D}=\left\{d_{\alpha}: \alpha \in \mathscr{A}\right\} \in \mathbb{J}_{(X, \mathscr{D})}$ and $\mathbb{J}_{(X, \mathscr{D})} \neq\{\mathscr{D}\}$.

(b) $\left(\left[7\right.\right.$, Remark 1.1]) Let $\mathscr{J}=\left\{J_{\alpha}: X^{2} \rightarrow[0 ; \infty), \alpha \in\right.$ $\mathscr{A}\} \in \mathbb{J}_{(X, \mathscr{D})}$. If $x \neq y, x, y \in X$, then $\exists_{\alpha \in \mathscr{A}}\left\{J_{\alpha}(x, y)>\right.$ $\left.0 \vee J_{\alpha}(y, x)>0\right\}$.

(c) Let $\mathscr{J}=\left\{J_{\alpha}: X^{2} \rightarrow[0 ; \infty), \alpha \in \mathscr{A}\right\} \in \mathbb{J}_{(X, \mathscr{D})}$. If $\forall_{\alpha \in \mathscr{A}} \forall_{x \in X}\left\{J_{\alpha}(x, x)=0\right\}$, then, for each $\alpha \in \mathscr{A}, J_{\alpha}$ is quasipseudometric; examples of $\mathscr{J}=\left\{J_{\alpha}: X^{2} \rightarrow\right.$ $[0 ; \infty), \alpha \in \mathscr{A}\} \in \mathbb{J}_{(X, \mathscr{D})}$ such that the maps $J_{\alpha}, \alpha \in \mathscr{A}$, are not quasipseudometrics are given in Section 4 .

Definition 4. Let $(X, \mathscr{D})$ be a Hausdorff uniform space. $\mathscr{J}=$ $\left\{J_{\alpha}: X^{2} \rightarrow[0 ; \infty), \alpha \in \mathscr{A}\right\} \in \mathbb{J}_{(X, \mathscr{D})}$ is said to be admissible if $X_{\mathcal{g}}^{0} \neq \varnothing$ where

$$
X_{\mathscr{g}}^{0}=\left\{x \in X: \forall_{\alpha \in \mathscr{A}}\left\{J_{\alpha}(x, x)=0\right\}\right\} .
$$

Remark 5. It is a remarkable fact that $\mathscr{D}$-family is admissible and $X_{\mathscr{D}}^{0}=X$. Indeed, we have that $X=X_{\mathcal{J}}^{0} \cup X_{\mathscr{g}}^{+}$, where $X_{\mathscr{J}}^{+}=$ $\left\{x \in X: \exists_{\alpha \in \mathscr{A}}\left\{J_{\alpha}(x, x)>0\right\}\right\}$. Therefore, by Definition 4, we get the following $X_{\mathscr{D}}^{0}=\left\{x \in X: \forall_{\alpha \in \mathscr{A}}\left\{d_{\alpha}(x, x)=0\right\}\right\}=X$.

Let $2^{X}$ denote the family of all nonempty subsets of a space $X$. A set-valued dynamic system is defined as a pair $(X, T)$, where $X$ is a certain space and $T$ is a set-valued map $T: X \rightarrow 2^{X}$; in particular, a set-valued dynamic system includes the usual dynamic system $(X, T)$ where $T: X \rightarrow X$ is a single-valued map.

Let $(X, T)$ be a set-valued dynamic system. By $\operatorname{Fix}(T)$ and $\operatorname{End}(T)$ we denote the sets of all fixed points and endpoints of $T$, respectively; that is, $\operatorname{Fix}(T)=\{w \in X: w \in T(w)\}$ and $\operatorname{End}(T)=\{w \in X:\{w\}=T(w)\}$. A dynamic process or a trajectory starting at $w_{0} \in X$ or a motion of the system $(X, T)$ at $w_{0}$ is a sequence $\left(w_{m}: m \in\{0\} \cup \mathbb{N}\right)$ defined by $w_{m} \in T\left(w_{m-1}\right)$ for $m \in \mathbb{N}$ (see, Aubin and Siegel [8], Aubin and Ekeland [9], Aubin and Frankowska [10], and Yuan [11]).

Recall that a map $\omega: X \rightarrow[0 ;+\infty]$ is proper if its effective domain, $\operatorname{dom}(\omega)=\{x: \omega(x) \neq+\infty\}$, is nonempty.

Caristi's fixed point theorem [12] concerning dissipative single-valued dynamic systems $(T, X)$ in metric spaces $(X, d)$ with lower semicontinuous entropies $\omega: X \rightarrow[0 ;+\infty]$ is equivalent to Ekeland's variational principle [13-15] providing approximate solutions of nonconvex minimization problems concerning lower semicontinuous maps $\omega: X \rightarrow$ $[0 ;+\infty]$.

Theorem 6. Let $(X, d)$ be a complete metric space.

(I) (Caristi [12]) Let $\omega: X \rightarrow[0 ;+\infty]$ be a map which is proper lower semicontinuous and let $(T, X)$ be a singlevalued dynamic system satisfying the condition

$$
\forall_{x \in X} \quad\{\omega(T(x))+d(x, T(x)) \leqslant \omega(x)\} .
$$

Then $\operatorname{Fix}(T) \neq \varnothing$ (i.e., there exists $w \in X$ such that $T(w)=w)$.

(II) (Ekeland [13-15]) Let $\omega: X \rightarrow[0 ;+\infty]$ be a map which is proper lower semicontinuous. For every $\varepsilon>0$ and for every $x_{0} \in \operatorname{dom}(\omega)$, there exists $u \in X$ such that $\omega(u)+\varepsilon d\left(x_{0}, u\right) \leqslant \omega\left(x_{0}\right)$ and $\forall_{x \in X \backslash\{u\}}\{\omega(u)<$ $\omega(x)+\varepsilon d(x, u)\}$.

Let $(X, \mathscr{D})$ be a sequentially complete uniform space. We say that a set $Y \in 2^{X}$ is closed in $X$ if $Y=\operatorname{cl}_{X}(Y)$, where $\operatorname{cl}_{X}(Y)$, the closure of $Y$ in $X$, denotes the set of all $w \in X$ for which there exists a sequence $\left(w_{m}: m \in \mathbb{N}\right)$ in $Y$ which converges to $w$. If a set $Y \in 2^{X}$ is closed in $X$, then $(Y, \mathscr{D})$ is a sequentially complete uniform space.

Define $\mathrm{Cl}(X)=\left\{Y \in 2^{X}: Y=\mathrm{cl}_{X}(Y)\right\}$; that is, $\mathrm{Cl}(X)$ denotes the class of all nonempty closed subsets of $X$.

The following fixed point and endpoint version of Caristi type theorem for dissipative set-valued dynamic systems without lower semicontinuous entropies in uniform spaces with structures determined by $\mathscr{J} \in \mathbb{I}_{(X, \mathscr{D})}$ is included in a more general result [6, Theorem 4.5].

Theorem 7. Let $(X, \mathscr{D})$ be a Hausdorff sequentially complete uniform space and let $\mathscr{J}=\left\{J_{\alpha}: X^{2} \rightarrow[0 ; \infty), \alpha \in \mathscr{A}\right\} \in$ $\mathbb{J}_{(X, \mathscr{D})}$ be admissible.

(I) (Fixed point theorem) Assume the following.

(A1) The family $\Omega=\left\{\omega_{\alpha}: X \rightarrow[0 ; \infty], \alpha \in \mathscr{A}\right\}$ satisfies $D_{\Omega}=\bigcap_{\alpha \in \mathscr{A}} \operatorname{dom}\left(\omega_{\alpha}\right) \neq \varnothing$.

(A2) $\Upsilon=\left\{\varepsilon_{\alpha}, \alpha \in \mathscr{A}\right\}$ is a family of finite positive numbers.

(A3) $(X, T)$ is a set-valued dynamic system.

(A4) For each $x \in X, Q_{\mathscr{g}, \Omega, \Upsilon ; T}(x)$ is a set defined by

$Q_{\mathscr{F}, \Omega, \Upsilon ; T}(x)$

$$
=\left\{y \in T(x) \cap X_{\mathscr{g}}^{0}: \forall_{\alpha \in \mathscr{A}}\left\{\omega_{\alpha}(y)+\varepsilon_{\alpha} J_{\alpha}(x, y) \leqslant \omega_{\alpha}(x)\right\}\right\} .
$$

(A5) For each $x \in X_{\mathscr{g}}^{0}$, the set $Q_{\mathscr{F}, \Omega, \Upsilon ; T}(x)$ is nonempty.

(A6) For each $x \in X_{\mathscr{f}}^{0}$, the set $Q_{\mathscr{F}, \Omega, \Upsilon ; T}(x)$ is a closed subset in $X$.

Then, there exists $w \in D_{\Omega} \cap X_{g}^{0}$ such that $w \in T(w)$ (i.e., $\forall_{\alpha \in \mathscr{A}}\left\{J_{\alpha}(w, w)=0\right\}$ and $\left.w \in \operatorname{Fix}(T)\right)$. 
(II) (Endpoint theorem) Assume, in addition, that

(A7) for each $x \in X_{q}^{0}$, each dynamic process $\left(w_{m}\right.$ : $m \in\{0\} \cup \mathbb{N})$ starting at $w_{0}=x$ and satisfying $\forall_{m \in\{0\} \cup \mathbb{N}}\left\{w_{m+1} \in T\left(w_{m}\right)\right\}$ satisfies $\forall_{m \in\{0\} \cup \mathbb{N}}\left\{w_{m+1} \in Q_{\mathcal{F}, \Omega, Y ; T}\left(w_{m}\right)\right\}$.

Then, there exists $w \in D_{\Omega} \cap X_{\mathcal{g}}^{0}$ such that $\{w\}=T(w)$ (i.e., $\forall_{\alpha \in \mathscr{A}}\left\{J_{\alpha}(w, w)=0\right\}$ and $w \in$ End $\left.(T)\right)$.

It is known that a weaker condition than continuity is lower semicontinuity.

Definition 8 . Let $(X, \mathscr{D})$ be a Hausdorff sequentially complete uniform space. Let $E \subseteq X, E \neq \varnothing$ and let $f: E \rightarrow[0 ; \infty]$. The map $f$ is lower semicontinuous on $E$ with respect to $X$ (written: $f$ is $(E, X)$-lsc when $E \neq X$ and $f$ is $l s c$ when $E=X$ ) if the set $\{y \in E: f(y) \leqslant c\}$ is a closed subset in $X$ for each $c \in[0 ; \infty)$.

The following alternative characterizations of lower semicontinuity hold.

Theorem 9. Let $(X, \mathscr{D})$ be a Hausdorff sequentially complete uniform space. Let $E \subseteq X, E \neq \varnothing$ and let $f: E \rightarrow[0 ; \infty]$. The following conditions are equivalent.

(Z1) The map $f$ is lower semicontinuous on $E$ with respect to $X$.

(Z2) For each $x_{0} \in E$,

$$
f\left(x_{0}\right) \leqslant \liminf _{x \rightarrow x_{0}, x \in X} f(x) ;
$$

here

$\liminf _{x \rightarrow x_{0}, x \in X} f(x)=\sup \left\{\inf \left\{f(x): x \in E \cap\left(U \backslash\left\{x_{0}\right\}\right)\right\}:\right.$

$$
\begin{gathered}
U \text { open in } X, x_{0} \in U, \\
\left.E \cap\left(U \backslash\left\{x_{0}\right\}\right) \neq \varnothing\right\} .
\end{gathered}
$$

(Z3) The map $f$ is sequentially lower semicontinuous on $E$ with respect to $X$; that is, for each $x_{0} \in E$,

$$
f\left(x_{0}\right) \leqslant \liminf _{m \rightarrow \infty} f\left(x_{m}\right)
$$

for any sequence $\left(x_{m}: m \in \mathbb{N}\right)$ in $X$ such that $\forall_{\alpha \in \mathscr{A}}\left\{\lim _{m \rightarrow \infty} d_{\alpha}\left(x_{m}, x_{0}\right)=0\right\}$; here

$$
\liminf _{m \rightarrow \infty} f\left(x_{m}\right)=\sup \left\{\inf \left\{f\left(x_{m}\right): m \geqslant n\right\}: n \in \mathbb{N}\right\} \text {. }
$$

Remark 10 (see [6, Remark 4.6]). The following hold.

(a) A special case of condition (A6) is a condition ( $\left.\mathrm{A}^{\prime}{ }^{\prime}\right)$ defined by

$\left(\mathrm{A}^{\prime}\right)$ for each $(x, \alpha) \in X_{\mathcal{g}}^{0} \times \mathscr{A}$, the map $\omega_{\alpha}(\cdot)+$ $\varepsilon_{\alpha} J_{\alpha}(x, \cdot): T(x) \cap X_{\mathscr{J}}^{0} \rightarrow[0 ; \infty]$ is $(T(x) \cap$ $\left.X_{\mathscr{J}}^{0}, X\right)$-lsc. (b) If $\mathscr{J}=\mathscr{D}$, then a special case of condition (A6) is a condition $\left(\mathrm{A} 6^{\prime \prime}\right)$ defined by

$\left(\mathrm{A} 6^{\prime \prime}\right)$ for each $(x, \alpha) \in X \times \mathscr{A}$, the map $\omega_{\alpha}(\cdot)+$ $\varepsilon_{\alpha} d_{\alpha}(x, \cdot): T(x) \rightarrow[0 ; \infty]$ is $(T(x), X)-1 s c$.

(c) Theorem 7(I) esentially generalizes Theorem 6(I) even in metric spaces.

A classic result of Banach [16], from 1922, is the milestone in the history of fixed point theory and its applications.

Theorem 11 (Banach [16]). Let $(X, d)$ be a complete metric space. Assume that the single-valued dynamic system $(X, T)$ is $(d, \lambda)$-contraction; that is,

$$
\exists_{\lambda \in[0 ; 1)} \forall_{x, y \in X} \quad\{d(T(x), T(y)) \leqslant \lambda d(x, y)\} .
$$

Then $T$ has a unique fixed point $w$ in $X$ (i.e., $T(w)=w$ and $\operatorname{Fix}(T)=\{w\})$ and, for each $w_{0} \in X$, the sequence $\left(w_{m}=\right.$ $\left.T^{[m]}\left(w_{0}\right): m \in \mathbb{N}\right)$ satisfies $\lim _{m \rightarrow \infty} d\left(w, w_{m}\right)=0$.

In a slightly different direction is the following elegant result of Nadler on set-valued dynamic systems.

Theorem 12 (Nadler [17, Theorem 5]). Let $(X, d)$ be a complete metric space, let $C B(X)$ denotes the class of all nonempty closed and bounded subsets of $X$, and let $H^{d}:(C B(X))^{2} \rightarrow$ $[0 ; \infty)$ be defined by

$$
\begin{aligned}
\forall_{A, B \in C B(X)} \quad H^{d} & (A, B) \\
& =\max \left\{\sup _{u \in A} d(u, B), \sup _{v \in B} d(v, A)\right\},
\end{aligned}
$$

where $\forall_{u \in X} \forall_{V \in C B(X)}\left\{d(u, V)=\inf _{z \in V} d(u, z)\right\}$. Assume that the set-valued dynamic system $(X, T)$ satisfying $T: X \rightarrow$ $C B(X)$ is $\left(H^{d}, \lambda\right)$-contraction; that is,

$$
\exists_{\lambda \in[0 ; 1)} \forall_{x, y \in X} \quad\left\{H^{d}(T(x), T(y)) \leqslant \lambda d(x, y)\right\} .
$$

Then $\operatorname{Fix}(T) \neq \varnothing$ (i.e., there exists $w \in X$ such that $w \in T(w))$.

Remark 13. Let $(X, d)$ be a complete metric space.

(a) It is well known that Caristi's fixed point theorem [12] yields Banach's [16] and Nadler's [17, Theorem 5] results.

(b) Maps $T:(X, d) \rightarrow(X, d)$ satisfying (3) are not necessarily continuous.

(c) It is well known that $\left(C B(X), H^{d}\right)$ is a complete metric space and that the continuity of maps $T:(X, d) \rightarrow$ $(X, d)$ and $T:(X, d) \rightarrow\left(C B(X), H^{d}\right)$ satisfying conditions (9) and (11) plays an important role in the proofs of Theorems 11 and 12, respectively.

Contractions (3) of Caristi, (9) of Banach, (11) of Nadler, and others are among the most important notions in fixed point theory, as well as in its numerous applications. As one will see from the literature, the known results about them 
have been achieved by employing complicated machineries from various branches of mathematics and the answers for many basic problems about them are still missing. Moreover, examples show that these fundamental results are not optimal even in metric spaces.

The several authors have made essential progress in these problems and have solved many cases, and similar methods and ideas have since been applied in greater generality; see for example [1-67] and the references cited therein. However, the complete solutions of some key open problems are still missing.

In this paper we show that there are complementary approaches to generalize the Nadler and Banach statements concerning uniform, locally convex, and metric spaces. They involve mixed properties of asymmetric structures and fixed point theory. One of the key ideas in this paper is that in $(X, \mathscr{D})$ the families $\mathscr{J}=\left\{J_{\alpha}: X^{2} \rightarrow[0 ; \infty), \alpha \in \mathscr{A}\right\} \in$ $\rrbracket_{(X, \mathscr{D})}$ construct the symmetric and asymmetric structures on $X$ which generalize the symmetric structure determined by $\mathscr{D}=\left\{d_{\alpha}: \alpha \in \mathscr{A}\right\}$ on $X$ and then, by subtle techniques, we may use stated above Theorem 7 .

More precisely, let $(X, \mathscr{D})$ be a Hausdorff uniform space. For $\mathscr{J}=\left\{J_{\alpha}: X^{2} \rightarrow[0 ; \infty), \alpha \in \mathscr{A}\right\} \in \mathbb{J}_{(X, \mathscr{D})}$ and $v \in\{1,2\}$, let the distance $\mathscr{H}_{v}^{\mathscr{J}}$ on $\mathrm{Cl}(X)$ be defined as in Definitions 15 and 23 , and let the distance $\mathscr{B}_{v}^{\mathscr{F}}$ on $X$ be defined as in Definitions 29 and 33.

This paper has two aims.

(1) To determine $\mathscr{J}=\left\{J_{\alpha}: X^{2} \rightarrow[0 ; \infty), \alpha \epsilon\right.$ $\mathscr{A}\} \in \mathbb{J}_{(X, \mathscr{D})}$, various classes of not necessarily continuous set-valued dynamic systems $(X, T)$ satisfying $T:(X, \mathscr{J}) \rightarrow\left(\mathrm{Cl}(X), \mathscr{H}_{v}^{\mathcal{F}}\right), v \in\{1,2\}$, and the conditions guaranteeing that the maps $x \rightarrow$ $\inf _{z \in T(x)} J_{\alpha}(x, z), \alpha \in \mathscr{A}$, attains its global optimal minimum at a point $w$ (not necessarily unique) satisfying $\forall_{\alpha \in \mathscr{A}}\left\{J_{\alpha}(w, T(w))=0\right\}$ and $w \in \operatorname{Fix}(T)$ or $w \in \operatorname{End}(T)$.

(2) To determine $\mathscr{J}=\left\{J_{\alpha}: X^{2} \rightarrow[0 ; \infty), \alpha \in \mathscr{A}\right\} \in$ $\mathbb{J}_{(X, \mathscr{D})}$, various classes of not necessarily continuous single-valued dynamic systems $(X, T)$ satisfying $T$ : $(X, \mathscr{J}) \rightarrow\left(X, \mathscr{B}_{v}^{\mathscr{F}}\right), v \in\{1,2\}$, and the conditions guaranteeing that the maps $x \rightarrow J_{\alpha}(x, T(x)), \alpha \in \mathscr{A}$, attains its unique global optimal approximate minimum at $w$ satisfying $J_{\alpha}(w, T(w))=0, \alpha \in \mathscr{A}, T(w)=$ $w$ and $\forall_{\alpha \in \mathscr{A}}\left\{\lim _{m \rightarrow \infty} J_{\alpha}\left(w, w_{m}\right)=\lim _{m \rightarrow \infty} J_{\alpha}\left(w_{m}\right.\right.$, $\left.w)=\lim _{m \rightarrow \infty} d_{\alpha}\left(w, w_{m}\right)=0\right\}$, where $\left(w_{m}=\right.$ $\left.T^{[m]}\left(w_{0}\right): m \in \mathbb{N}\right)$ and $w_{0} \in X$ is arbitrary.

Remark 14. (a) The methods of this paper provide a way to compute the fixed point and endpoint theorems in uniform, locally convex and metric spaces with structures determined by $\mathscr{J}=\left\{J_{\alpha}: X^{2} \rightarrow[0 ; \infty), \alpha \in \mathscr{A}\right\} \in \mathbb{J}_{(X, \mathscr{D})}$.

(b) Theorems 17, 20, 21, 22, 25, 26, 27, 31, 34 and Examples 1-4 and 5-7 shows that our fixed point and endpoint results are new in uniform and locally convex spaces and even in metric spaces, are different from fixed point and endpoint results given in the literature, and their proofs are simpler.

\section{Fixed Point and Endpoint Theorems for Set-Valued Contractions (of Nadler Type) in Uniform and Metric Spaces}

The following definitions will be much used in the sequel.

Definition 15 . Let $(X, \mathscr{D})$ be a Hausdorff sequentially complete uniform space, assume that $\mathscr{J}=\left\{J_{\alpha}: X^{2} \rightarrow[0 ; \infty)\right.$, $\alpha \in \mathscr{A}\} \in \mathbb{J}_{(X, \mathscr{D})}$, let

$$
\forall_{\alpha \in \mathscr{A}} \forall_{u \in X} \forall_{V \in \mathrm{Cl}(X)} \quad\left\{J_{\alpha}(u, V)=\inf \left\{J_{\alpha}(u, z): z \in V\right\}\right\},
$$

and let $v \in\{1,2\}$.

(a) Define on $\mathrm{Cl}(X)$ the distance $\mathscr{H}_{v}^{\mathcal{J}}, \mathscr{H}_{v}^{\mathcal{F}}=\left\{H_{v ; \alpha}^{\mathscr{J}}\right.$ : $\left.\mathrm{Cl}(X)^{2} \rightarrow[0 ; \infty], \alpha \in \mathscr{A}\right\}$, as follows:

$\forall_{\alpha \in \mathscr{A}} \forall_{A, B \in \mathrm{Cl}(X)}$

$H_{v ; \alpha}^{\mathcal{g}}(A, B)=\max \left\{\sup _{u \in A} J_{\alpha}(u, B), \sup _{z \in B} J_{\alpha}(z, A)\right\} \quad$ if $v=1$,

$\forall_{\alpha \in \mathscr{A}} \forall_{A, B \in \mathrm{Cl}(X)} \quad\left\{H_{v ; \alpha}^{\mathscr{\mathcal { F }}}(A, B)=\sup _{u \in A} J_{\alpha}(u, B)\right\} \quad$ if $v=2$.

(b) Let a set-valued dynamic system $(X, T)$ satisfy $T$ : $X \rightarrow \mathrm{Cl}(X)$. If $(X, T)$ satisfies

$\forall_{\alpha \in \mathscr{A}} \exists_{\lambda_{\alpha} \in[0 ; 1)} \forall_{x, y \in X} \quad\left\{H_{v ; \alpha}^{\mathcal{g}}(T(x), T(y)) \leqslant \lambda_{\alpha} J_{\alpha}(x, y)\right\}$,

then we say that $(X, T)$ is a $\left(\mathscr{H}_{v}^{\mathscr{g}}, \Lambda\right)$-contraction on $X$ for $\Lambda=\left\{\lambda_{\alpha} \in[0 ; 1), \alpha \in \mathscr{A}\right\}$.

Remark 16. Each $\left(\mathscr{H}_{1}^{\mathscr{g}}, \Lambda\right)$-contraction on $X$ is $\left(\mathscr{H}_{2}^{\mathscr{g}}, \Lambda\right)$ contraction on $X$ but converse does not hold.

One can prove the following characterizations of $\left(\mathscr{H}_{v}^{\mathcal{g}}, \Lambda\right)$-contractions $(X, T)$ :

Theorem 17. Let $(X, \mathscr{D})$ be a Hausdorff sequentially complete uniform space, $\mathscr{J}=\left\{J_{\alpha}: X^{2} \rightarrow[0 ; \infty), \alpha \in \mathscr{A}\right\} \in \mathbb{J}_{(X, \mathscr{D})}$ and $v \in\{1,2\}$. Suppose also the following.

(I) A set-valued dynamic system $(X, T)$ satisfies $T: X \rightarrow$ $\mathrm{Cl}(X)$.

(II) There exists a family $\Lambda=\left\{\lambda_{\alpha} \in[0 ; 1), \alpha \in \mathscr{A}\right\}$ such that $(X, T)$ is a $\left(\mathscr{H}_{v}^{\mathcal{F}}, \Lambda\right)$-contraction on $X$.

(III) The family $\Gamma=\left\{\gamma_{\alpha} \in(0 ; 1), \alpha \in \mathscr{A}\right\}$ satisfies $\forall_{\alpha \in \mathscr{A}}\left\{\lambda_{\alpha}<\gamma_{\alpha}\right\}$.

The following hold.

(B1) $\forall_{\alpha \in \mathscr{A}} \forall_{x \in X}\left\{\left\{y \in T(x): J_{\alpha}(y, T(y)) \leqslant \lambda_{\alpha} J_{\alpha}(x, y)\right\}=\right.$ $T(x)\}$.

(B2) $\forall_{\alpha \in \mathscr{A}} \forall_{x \in X}\left\{U_{\Gamma, \alpha}(x) \neq \varnothing\right\}$ where

$$
\begin{array}{r}
U_{\Gamma, \alpha}(x)=\left\{y \in T(x): \gamma_{\alpha} J_{\alpha}(x, y) \leqslant J_{\alpha}(x, T(x))\right\}, \\
\alpha \in \mathscr{A}, \quad x \in X .
\end{array}
$$


(B3) $\forall_{\alpha \in \mathscr{A}} \forall_{x \in X}\left\{U_{\Gamma, \alpha}(x) \subset V_{\Gamma, \alpha}(x)\right\}$ where

$$
\begin{gathered}
V_{\Gamma, \alpha}(x)=\left\{y \in T(x): J_{\alpha}(y, T(y))+\left(\gamma_{\alpha}-\lambda_{\alpha}\right) J_{\alpha}(x, y)\right. \\
\left.\leqslant J_{\alpha}(x, T(x))\right\}, \quad \alpha \in \mathscr{A}, \quad x \in X .
\end{gathered}
$$

(B4) $\forall_{\alpha \in \mathscr{A}} \forall_{x \in X} \forall y \in T(x)\left\{0 \leqslant J_{\alpha}(x, T(x))-J_{\alpha}(y, T(y)) \leqslant(1+\right.$ $\left.\left.\lambda_{\alpha}\right) J_{\alpha}(x, y)\right\}$.

(B5) $\forall_{\alpha \in \mathscr{A}} \forall_{x \in X} \forall_{y \in T(x)}\left\{J_{\alpha}(y, T(y))+J_{\alpha}(x, y) \geqslant J_{\alpha}(x\right.$, $T(x))\}$.

Proof. Let the family $\Omega^{\mathscr{F}}=\left\{\omega_{\alpha}^{\mathscr{F}}: X \rightarrow[0 ; \infty), \alpha \in \mathscr{A}\right\}$ be defined by

$$
\forall_{\alpha \in \mathscr{A}} \forall_{x \in X} \quad\left\{\omega_{\alpha}^{\mathcal{F}}(x)=J_{\alpha}(x, T(x))\right\} .
$$

Proof of (B1). By assumption (II) and Definitions 15(a) and 15(b),

$$
\begin{aligned}
& \forall_{\alpha \in \mathscr{A}} \forall_{x, y \in X}\left\{\sup _{u \in T(x)} J_{\alpha}(u, T(y))\right. \\
& \leqslant \max \left\{\sup _{u \in T(x)} J_{\alpha}(u, T(y)),\right. \\
& \left.\sup _{z \in T(y)} J_{\alpha}(z, T(x))\right\} \\
& \left.\forall \lambda_{\alpha \in \mathscr{A}} J_{\alpha, y \in X}(x, y)\right\} \text { if } v=1, \\
& \left\{\sup _{u \in T(x)} J_{\alpha}(u, T(y)) \leqslant \lambda_{\alpha} J_{\alpha}(x, y)\right\} \quad \text { if } v=2 .
\end{aligned}
$$

Using this, we may thus conclude that

$$
\begin{gathered}
\forall_{\alpha \in \mathscr{A}} \forall_{x \in X} \forall_{y \in T(x)} \quad\left\{\omega_{\alpha}^{\mathcal{J}}(y) \leqslant \sup _{u \in T(x)} J_{\alpha}(u, T(y))\right. \\
\left.\leqslant \lambda_{\alpha} J_{\alpha}(x, y)\right\}
\end{gathered}
$$

and hence

$$
\forall_{\alpha \in \mathscr{A}} \forall_{x \in X} \quad\left\{T(x) \subset\left\{y \in T(x): \omega_{\alpha}^{\mathscr{F}}(y) \leqslant \lambda_{\alpha} J_{\alpha}(x, y)\right\}\right\} .
$$

On the other hand it is clear that

$$
\forall_{\alpha \in \mathscr{A}} \forall_{x \in X} \quad\left\{\left\{y \in T(x): \omega_{\alpha}^{\mathcal{J}}(y) \leqslant \lambda_{\alpha} J_{\alpha}(x, y)\right\} \subset T(x)\right\} .
$$

By applying (20) and (21), we obtain (B1).
Proof of (B2). By (12), we have

$$
\forall_{\alpha \in \mathscr{A}} \forall_{x \in X} \quad\left\{\omega_{\alpha}^{\mathcal{g}}(x)=\inf _{y \in T(x)} J_{\alpha}(x, y)\right\} .
$$

Further, by assumption (III), $\forall_{\alpha \in \mathscr{A}}\left\{\gamma_{\alpha} \in(0 ; 1)\right\}$. Hence, for arbitrary and fixed $\alpha \in \mathscr{A}$ and $x \in X$, by (22) and definition of infimum, we obtain that

$$
\exists_{y_{0} \in T(x)} \quad\left\{\gamma_{\alpha} J_{\alpha}\left(x, y_{0}\right) \leqslant \inf _{y \in T(x)} J_{\alpha}(x, y)=\omega_{\alpha}^{\mathcal{g}}(x)\right\} .
$$

Consequently,

$$
\forall_{\alpha \in \mathscr{A}} \forall_{x \in X} \exists_{y_{0} \in T(x)} \quad\left\{y_{0} \in U_{\Gamma, \alpha}(x)\right\} .
$$

So we have proved (B2).

Proof of (B3). Let $\alpha \in \mathscr{A}, x \in X$, and $y_{0} \in U_{\Gamma, \alpha}(x)$ be arbitrary and fixed. Then, by (B2), we have $y_{0} \in T(x)$ and

$$
\gamma_{\alpha} J_{\alpha}\left(x, y_{0}\right) \leqslant \omega_{\alpha}^{\mathcal{g}}(x)
$$

Clearly, by (B1), property $y_{0} \in T(x)$ implies $\omega_{\alpha}^{\mathcal{F}}\left(y_{0}\right) \leqslant$ $\lambda_{\alpha} J_{\alpha}\left(x, y_{0}\right)$. Thus

$$
-\lambda_{\alpha} J_{\alpha}\left(x, y_{0}\right) \leqslant-\omega_{\alpha}^{\mathcal{g}}\left(y_{0}\right) .
$$

Using (25) and (26) we obtain

$$
\left(\gamma_{\alpha}-\lambda_{\alpha}\right) J_{\alpha}\left(x, y_{0}\right) \leqslant \omega_{\alpha}^{\mathcal{F}}(x)-\omega_{\alpha}^{\mathcal{J}}\left(y_{0}\right) .
$$

We proved that

$$
\begin{aligned}
& \forall_{\alpha \in \mathscr{A}} \forall_{x \in X} \quad\left\{U _ { \Gamma , \alpha } ( x ) \subset \left\{y \in T(x):\left(\gamma_{\alpha}-\lambda_{\alpha}\right) J_{\alpha}(x, y)\right.\right. \\
& \left.\left.\leqslant \omega_{\alpha}^{\mathcal{F}}(x)-\omega_{\alpha}^{\mathcal{F}}(y)\right\}\right\} \text {. }
\end{aligned}
$$

Therefore, (B3) holds.

Proof of (B4). Let $\alpha \in \mathscr{A}, x \in X$, and $y \in T(x)$ be arbitrary and fixed. Then, by (B1), since $y \in T(x)$, we obtain $\omega_{\alpha}^{\mathscr{g}}(y) \leqslant$ $\lambda_{\alpha} J_{\alpha}(x, y)$. This and (22) imply

$$
-\omega_{\alpha}^{\mathcal{g}}(y) \geqslant-\lambda_{\alpha} J_{\alpha}(x, y) \geqslant-\lambda_{\alpha} \omega_{\alpha}^{\mathcal{g}}(x) \geqslant-\omega_{\alpha}^{\mathcal{F}}(x) .
$$

Therefore,

$$
\forall_{\alpha \in \mathscr{A}} \forall_{x \in X} \forall_{y \in T(x)} \quad\left\{0 \leqslant \omega_{\alpha}^{\mathcal{g}}(x)-\omega_{\alpha}^{\mathcal{g}}(y)\right\}
$$

holds. Next, it follows from (22) and (B1) that

$$
\begin{gathered}
\forall_{\alpha \in \mathscr{A}} \forall_{x \in X} \forall_{y \in T(x)} \quad\left\{\omega_{\alpha}^{\mathcal{g}}(x)-\omega_{\alpha}^{\mathcal{g}}(y) \leqslant \omega_{\alpha}^{\mathcal{g}}(x)+\omega_{\alpha}^{\mathcal{g}}(y)\right. \\
\left.\leqslant\left(1+\lambda_{\alpha}\right) J_{\alpha}(x, y)\right\} .
\end{gathered}
$$

This shows that (B4) holds.

Proof of (B5). By (30) and (22),

$\forall_{\alpha \in \mathscr{A}} \forall_{x \in X} \forall_{y \in T(x)} \quad\left\{\omega_{\alpha}^{\mathscr{g}}(x)-\omega_{\alpha}^{\mathscr{g}}(y) \leqslant \omega_{\alpha}^{\mathscr{g}}(x) \leqslant J_{\alpha}(x, y)\right\}$.

Therefore, (B5) holds. 
Definition 18. Let $(X, \mathscr{D})$ be a Hausdorff sequentially complete uniform space and let $\mathscr{J}=\left\{J_{\alpha}: X^{2} \rightarrow[0 ; \infty), \alpha \in\right.$ $\mathscr{A}\} \in \mathbb{J}_{(X, \mathscr{D})}$. We say that the family $\mathscr{J}$ is continuous in $X$ if, for each $x_{0} \in X$ and for each sequence $\left(x_{m}: m \in \mathbb{N}\right)$ in $X$ such that

$$
\forall_{\alpha \in \mathscr{A}} \quad\left\{\lim _{m \rightarrow \infty} d_{\alpha}\left(x_{m}, x_{0}\right)=0\right\}
$$

we have

$$
\forall_{\alpha \in \mathscr{A}} \quad\left\{\liminf _{m \rightarrow \infty} J_{\alpha}\left(x_{m}, x_{0}\right)=\liminf _{m \rightarrow \infty} J_{\alpha}\left(x_{0}, x_{m}\right)=0\right\} .
$$

Remark 19. The family $\mathscr{D}$ is continuous in $X$.

Assertion (B5) says that, for each $x \in X$, the set

$$
\begin{aligned}
Q_{\mathscr{g} ; T}(x)=\left\{y \in T(x): \forall_{\alpha \in \mathscr{A}}\right. & \left\{J_{\alpha}(y, T(y))\right. \\
& \left.\left.+J_{\alpha}(x, y) \geqslant J_{\alpha}(x, T(x))\right\}\right\}
\end{aligned}
$$

has the property

$$
\varnothing \neq Q_{\mathscr{F} ; T}(x)=T(x) \in \mathrm{Cl}(X) .
$$

Let $\Upsilon=\left\{\varepsilon_{\alpha}, \alpha \in \mathscr{A}\right\}$ be a family of positive numbers satisfying $\forall_{\alpha \in \mathscr{A}}\left\{\varepsilon_{\alpha} \in(0 ; 1)\right\}$ and, for each $x \in X$, let the the set $Q_{\mathscr{g}, Y ; T}(x)$ be defined by

$$
\begin{aligned}
Q_{\mathscr{f}, \Upsilon ; T}(x)= & \left\{y \in T(x) \cap X_{\mathcal{F}}^{0}:\right. \\
& \forall_{\alpha \in \mathscr{A}}\left\{J_{\alpha}(y, T(y))\right. \\
& \left.\left.\quad+\varepsilon_{\alpha} J_{\alpha}(x, y) \leqslant J_{\alpha}(x, T(x))\right\}\right\} .
\end{aligned}
$$

Now, for $\left(\mathscr{H}_{v}^{\mathscr{J}}, \Lambda\right)$-contractions $(X, T)$, we can give the following characterizations of the sets $Q_{\mathscr{F}, \Upsilon ; T}(x), x \in X$, defined in (37).

Theorem 20. Let $(X, \mathscr{D})$ be a Hausdorff sequentially complete uniform space, $\mathscr{J}=\left\{J_{\alpha}: X^{2} \rightarrow[0 ; \infty), \alpha \in \mathscr{A}\right\} \in \mathbb{J}_{(X, \mathscr{D})}$ and $v \in\{1,2\}$. Suppose also the following.

(I) $\mathcal{J}$ is admissible.

(II) A set-valued dynamic system $(X, T)$ satisfies $T: X \rightarrow$ $\mathrm{Cl}(X)$.

(III) There exists a family $\Lambda=\left\{\lambda_{\alpha} \in[0 ; 1), \alpha \in \mathscr{A}\right\}$ such that $(X, T)$ is a $\left(\mathscr{H}_{v}^{\mathscr{J}}, \Lambda\right)$-contraction on $X$.

(IV) For each family $\Gamma=\left\{\gamma_{\alpha} \in(0 ; 1), \alpha \in \mathscr{A}\right\}$ satisfying $\forall_{\alpha \in \mathscr{A}}\left\{\lambda_{\alpha}<\gamma_{\alpha}\right\}$ and for each $x \in X$, let the set $\mathrm{Q}_{\mathscr{f}, \Gamma-\Lambda ; T}(x)$ be defined by

$$
\begin{aligned}
Q_{\mathscr{g}, \Gamma-\Lambda ; T}(x)=\{y \in T(x) & \cap X_{\mathcal{g}}^{0}: \\
\forall_{\alpha \in \mathscr{A}}\{ & J_{\alpha}(y, T(y)) \\
+ & \left(\gamma_{\alpha}-\lambda_{\alpha}\right) J_{\alpha}(x, y) \\
\leqslant & \left.\left.J_{\alpha}(x, T(x))\right\}\right\} .
\end{aligned}
$$

The following hold.

(C1) If there exists a family $\Gamma^{0}=\left\{\gamma_{\alpha}^{0} \in(0 ; 1), \alpha \in \mathscr{A}\right\}$ satisfying $\forall_{\alpha \in \mathscr{A}}\left\{\lambda_{\alpha}<\gamma_{\alpha}^{0}\right\}$ and such that

$$
\begin{aligned}
& \forall_{x \in X_{\mathcal{f}}^{0}} \quad\{\{y \in T(x) \cap X_{\mathcal{g}}^{0}: \\
& \forall_{\alpha \in \mathscr{A}} \quad\left\{\gamma_{\alpha}^{0} J_{\alpha}(x, y)\right. \\
&\left.\left.\left.\quad \leqslant J_{\alpha}(x, T(x))\right\}\right\} \neq \varnothing\right\},
\end{aligned}
$$

then $\forall_{x \in X_{\mathscr{f}}^{0}}\left\{Q_{\mathscr{g}, \Gamma^{0}-\Lambda ; T}(x) \neq \varnothing\right\}$.

(C2) If there exists a family $\Gamma^{0}=\left\{\gamma_{\alpha}^{0} \in(0 ; 1), \alpha \in \mathscr{A}\right\}$ satisfying $\forall_{\alpha \in \mathscr{A}}\left\{\lambda_{\alpha}<\gamma_{\alpha}^{0}\right\}$ and such that, for each $(x, \alpha) \in X_{\mathcal{g}}^{0} \times \mathscr{A}$, the map

$$
J_{\alpha}(\cdot, T(\cdot))+\left(\gamma_{\alpha}^{0}-\lambda_{\alpha}\right) J_{\alpha}(x, \cdot): T(x) \cap X_{\mathcal{J}}^{0} \rightarrow[0 ; \infty)
$$

is $\left(T(x) \cap X_{\mathcal{g}}^{0}, X\right)$-lsc, then, for each $x \in X_{\mathcal{g}}^{0}$, $Q_{\mathscr{F}, \Gamma^{0}-\Lambda ; T}(x)$ is a closed subset in $X$.

(C3) Let the family $\mathcal{J}$ be continuous in $X$. Then, for each family $\Gamma=\left\{\gamma_{\alpha} \in(0 ; 1), \alpha \in \mathscr{A}\right\}$ satisfying $\forall_{\alpha \in \mathscr{A}}\left\{\lambda_{\alpha}<\right.$ $\left.\gamma_{\alpha}\right\}$ and for each $x \in X_{\mathscr{f}}^{0}, Q_{\mathscr{F}, \Gamma-\Lambda ; T}(x)$ is a closed subset in $X$.

(C4) Let $\mathcal{J}=\mathscr{D}$. If there exists a family $\Gamma^{0}=\left\{\gamma_{\alpha}^{0} \in\right.$ $(0 ; 1), \alpha \in \mathscr{A}\}$ satisfying $\forall_{\alpha \in \mathscr{A}}\left\{\lambda_{\alpha}<\gamma_{\alpha}^{0}\right\}$ and such that

$$
\begin{aligned}
\forall_{x \in X} \quad\{\{ & \{y \in T(x): \\
& \left.\left.\forall_{\alpha \in \mathscr{A}}\left\{\gamma_{\alpha}^{0} d_{\alpha}(x, y) \leqslant d_{\alpha}(x, T(x))\right\}\right\} \neq \varnothing\right\},
\end{aligned}
$$

then $\forall_{x \in X}\left\{Q_{\mathscr{D}, \Gamma^{0}-\Lambda ; T}(x) \neq \varnothing\right\}$.

(C5) Let $\mathscr{J}=\mathscr{D}$. Then, for each family $\Gamma=\left\{\gamma_{\alpha} \in(0 ; 1), \alpha \in\right.$ A\} satisfying $\forall_{\alpha \in \mathscr{A}}\left\{\lambda_{\alpha}<\gamma_{\alpha}\right\}$ and for each $x \in X$, $Q_{\mathscr{D}, \Gamma-\Lambda ; T}(x)$ is a closed subset in $X$.

Proof. Let the family $\Omega^{\mathcal{F}}=\left\{\omega_{\alpha}^{\mathcal{F}}: X \rightarrow[0 ; \infty), \alpha \in \mathscr{A}\right\}$ be defined by

$$
\forall_{\alpha \in \mathscr{A}} \forall_{x \in X} \quad\left\{\omega_{\alpha}^{\mathcal{g}}(x)=J_{\alpha}(x, T(x))\right\} .
$$

Proof of (C1). Denote

$$
\begin{aligned}
& \forall_{x \in X} \quad\left\{U_{\Gamma^{0}, \mathscr{A}}(x)=\bigcap_{\alpha \in \mathscr{A}} U_{\Gamma^{0}, \alpha}(x)\right\}, \\
& \forall_{x \in X} \quad\left\{V_{\Gamma^{0}, \mathscr{A}}(x)=\bigcap_{\alpha \in \mathscr{A}} V_{\Gamma^{0}, \alpha}(x)\right\} .
\end{aligned}
$$

Then, by (B2), (B3), and (IV),

$$
\begin{gathered}
\forall_{x \in X_{\mathcal{F}}^{0}} \quad\left\{U_{\Gamma^{0}, \mathscr{A}}(x) \cap X_{\mathcal{F}}^{0} \subset V_{\Gamma^{0}, \mathscr{A}}(x) \cap X_{\mathcal{F}}^{0}\right. \\
\left.=Q_{\mathscr{f}, \Gamma^{0}-\Lambda ; T}(x)\right\} .
\end{gathered}
$$


Hence, we conclude that, for each $x \in X_{\mathscr{y}}^{0}$, the set $Q_{\mathscr{F}, \Gamma^{0}-\Lambda ; T}(x)$ is nonempty whenever $\forall_{x \in X_{\mathscr{f}}^{0}}\left\{U_{\Gamma^{0}, \mathscr{A}}(x) \cap X_{\mathscr{J}}^{0} \neq\right.$ $\varnothing\}$.

Proof of (C2). The assertion follows immediately from Remark 10(a).

Proof of (C3). The assertion also follows from Remark 10(a). Indeed, let $x_{0} \in X$ be arbitrary and fixed and let a sequence $\left(x_{m}: m \in \mathbb{N}\right)$ in $X$ be convergent to $x_{0}$; that is, let $\forall_{\alpha \in \mathscr{A}}\left\{\lim _{m \rightarrow \infty} d_{\alpha}\left(x_{0}, x_{m}\right)=0\right\}$.

If $m \in \mathbb{N}, z \in T\left(x_{m}\right)$ and $\alpha \in \mathscr{A}$ are arbitrary and fixed, then, by $(\mathscr{F} 1)$,

$$
\begin{aligned}
& \forall_{u \in T\left(x_{0}\right)} \quad\left\{\omega_{\alpha}^{g}\left(x_{0}\right)=J_{\alpha}\left(x_{0}, T\left(x_{0}\right)\right) \leqslant J_{\alpha}\left(x_{0}, u\right)\right. \\
& \left.\leqslant J_{\alpha}\left(x_{0}, x_{m}\right)+J_{\alpha}\left(x_{m}, z\right)+J_{\alpha}(z, u)\right\} \text {. }
\end{aligned}
$$

This gives

$$
\omega_{\alpha}^{q}\left(x_{0}\right) \leqslant J_{\alpha}\left(x_{0}, x_{m}\right)+J_{\alpha}\left(x_{m}, z\right)+J_{\alpha}\left(z, T\left(x_{0}\right)\right) .
$$

Hence

$$
\omega_{\alpha}^{\mathcal{F}}\left(x_{0}\right) \leqslant J_{\alpha}\left(x_{0}, x_{m}\right)+J_{\alpha}\left(x_{m}, z\right)+\sup _{u \in T\left(x_{m}\right)} J_{\alpha}\left(u, T\left(x_{0}\right)\right) .
$$

Furthermore, this holds for each $z \in T\left(x_{m}\right)$ and, thus, by (12),

$$
\omega_{\alpha}^{g}\left(x_{0}\right) \leqslant J_{\alpha}\left(x_{0}, x_{m}\right)+\omega_{\alpha}^{g}\left(x_{m}\right)+\sup _{u \in T\left(x_{m}\right)} J_{\alpha}\left(u, T\left(x_{0}\right)\right) .
$$

However, $(X, T)$ is $\left(\mathscr{H}_{v}^{\mathcal{F}}, \Lambda\right)$-contraction on $X$. Therefore,

$$
\begin{aligned}
& \sup _{u \in T\left(x_{m}\right)} J_{\alpha}\left(u, T\left(x_{0}\right)\right) \\
& \leqslant \max \left\{\sup _{u \in T\left(x_{m}\right)} J_{\alpha}\left(u, T\left(x_{0}\right)\right), \sup _{z \in T\left(x_{0}\right)} J_{\alpha}\left(z, T\left(x_{m}\right)\right)\right\} \\
& \leqslant \lambda_{\alpha} J_{\alpha}\left(x_{m}, x_{0}\right) \quad \text { if } v=1, \\
& \sup _{u \in T\left(x_{m}\right)} J_{\alpha}\left(u, T\left(x_{0}\right)\right) \leqslant \lambda_{\alpha} J_{\alpha}\left(x_{m}, x_{0}\right) \quad \text { if } v=2 .
\end{aligned}
$$

Consequently, we obtain that

$$
\begin{aligned}
\omega_{\alpha}^{\mathcal{F}}\left(x_{0}\right) & \leqslant J_{\alpha}\left(x_{0}, x_{m}\right)+\omega_{\alpha}^{\mathcal{F}}\left(x_{m}\right)+\sup _{u \in T\left(x_{m}\right)} J_{\alpha}\left(u, T\left(x_{0}\right)\right) \\
& \leqslant J_{\alpha}\left(x_{0}, x_{m}\right)+\omega_{\alpha}^{\mathcal{F}}\left(x_{m}\right)+\lambda_{\alpha} J_{\alpha}\left(x_{m}, x_{0}\right) .
\end{aligned}
$$

Since the family $\mathscr{f}$ is continuous, this implies

$$
\omega_{\alpha}^{\mathcal{F}}\left(x_{0}\right) \leqslant \liminf _{m \rightarrow \infty} \omega_{\alpha}^{\mathcal{g}}\left(x_{m}\right) .
$$

Therefore, for each $\alpha \in \mathscr{A}, \omega_{\alpha}^{\mathcal{f}}(\cdot)$ is lsc in $X$.
Moreover, if $m \in \mathbb{N}, x \in X$, and $\alpha \in \mathscr{A}$ are arbitrary and fixed, then, by $(\mathscr{F} 1)$,

$$
J_{\alpha}\left(x, x_{0}\right) \leqslant J_{\alpha}\left(x, x_{m}\right)+J_{\alpha}\left(x_{m}, x_{0}\right) .
$$

Since $\mathcal{F}$ is continuous, this gives

$$
J_{\alpha}\left(x, x_{0}\right) \leqslant \liminf _{m \rightarrow \infty} J_{\alpha}\left(x, x_{m}\right) .
$$

that is, for each $(x, \alpha) \in X \times \mathscr{A}$, the map $J_{\alpha}(x, \cdot)$ is lsc in $X$.

Using these two facts, in particular, we have that, for each $(x, \alpha) \in X_{\mathscr{f}}^{0} \times \mathscr{A}$, the map

$$
\omega_{\alpha}^{\mathscr{g}}(\cdot)+\left(\gamma_{\alpha}-\lambda_{\alpha}\right) J_{\alpha}(x, \cdot): T(x) \cap X_{\mathscr{g}}^{0} \longrightarrow[0 ; \infty]
$$

is $\left(T(x) \cap X_{\mathscr{f}}^{0}, X\right)$-lsc; that is, $\left(\mathrm{A}^{\prime}\right)$ holds.

Proof of (C4). This follows from (C1).

Proof of (C5). This follows from (C3) and Remarks 3(a) and 19.

We use notations and auxiliary Theorems 17 and 20 above in proving the following basic fixed point and endpoint theorem for set-valued contractions with respect to $\mathscr{J} \in$ $\rrbracket_{(X, \mathscr{D})}$ (of Nadler-type) in uniform spaces $(X, \mathscr{D})$.

Theorem 21. Let $(X, \mathscr{D})$ be a Hausdorff sequentially complete uniform space, $\mathscr{J}=\left\{J_{\alpha}: X^{2} \rightarrow[0 ; \infty), \alpha \in \mathscr{A}\right\} \in \mathbb{J}_{(X, \mathscr{D})}$ and $v \in\{1,2\}$. Suppose also the following.

(I) $\mathcal{J}$ is admissible.

(II) A set-valued dynamic system $(X, T)$ satisfies $T: X \rightarrow$ $\mathrm{Cl}(X)$.

(III) There exists a family $\Lambda=\left\{\lambda_{\alpha} \in[0 ; 1), \alpha \in \mathscr{A}\right\}$ such that $(X, T)$ is a $\left(\mathscr{H}_{v}^{\mathcal{F}}, \Lambda\right)$-contraction on $X$.

(IV) For each family $\Gamma=\left\{\gamma_{\alpha} \in(0 ; 1), \alpha \in \mathscr{A}\right\}$ satisfying $\forall_{\alpha \in \mathscr{A}}\left\{\lambda_{\alpha}<\gamma_{\alpha}\right\}$ and for each $x \in X$, let the set $Q_{f, \Gamma-\Lambda ; T}(x)$ be defined by

$$
\begin{aligned}
& Q_{\mathscr{F}, \Gamma-\Lambda ; T}(x) \\
& \begin{aligned}
=\left\{y \in T(x) \cap X_{\mathscr{g}}^{0}: \forall_{\alpha \in \mathscr{A}}\left\{J_{\alpha}\right.\right. & (y, T(y)) \\
& +\left(\gamma_{\alpha}-\lambda_{\alpha}\right) J_{\alpha}(x, y) \\
& \left.\left.\leqslant J_{\alpha}(x, T(x))\right\}\right\} .
\end{aligned}
\end{aligned}
$$

(V) There exists a family $\Gamma^{0}=\left\{\gamma_{\alpha}^{0} \in(0 ; 1), \alpha \in \mathscr{A}\right\}$ satisfying $\forall_{\alpha \in \mathscr{A}}\left\{\lambda_{\alpha}<\gamma_{\alpha}^{0}\right\}$ and such that, for each $x \in X_{\mathscr{f}}^{0}, Q_{\mathscr{f}, \Gamma^{0}-\Lambda ; T}(x)$ is a nonempty closed subset in $X$.

The following hold,

(E1) (Fixed point theorem) $\operatorname{Fix}(T) \neq \varnothing$ and there exists $w \in \operatorname{Fix}(T)$ satisfying $\forall_{\alpha \in \mathscr{A}}\left\{J_{\alpha}(w, w)=0\right\}$. 
(E2) (Endpoint theorem) If, for each $x \in X_{g}^{0}$, each dynamic process $\left(w_{m}: m \in\{0\} \cup \mathbb{N}\right)$ starting at $w_{0}=$ $x$ and satisfying $\forall_{m \in\{0\} \cup \mathbb{N}}\left\{w_{m+1} \in T\left(w_{m}\right)\right\}$ satisfies $\forall_{m \in\{0\} \cup \mathbb{N}}\left\{w_{m+1} \in Q_{\mathscr{F}, \Gamma^{0}-\Lambda ; T}\left(w_{m}\right)\right\}$, then End $(T) \neq \varnothing$ and $\forall_{w \in \text { End }(T)} \forall_{\alpha \in \mathscr{A}}\left\{J_{\alpha}(w, w)=0\right\}$.

Proof. The proof will be broken into five steps.

Step 1. Let the family $\Omega^{\mathscr{F}}=\left\{\omega_{\alpha}^{\mathscr{F}}: X \rightarrow[0 ; \infty), \alpha \in \mathscr{A}\right\}$ be defined by

$$
\forall_{\alpha \in \mathscr{A}} \forall_{x \in X} \quad\left\{\omega_{\alpha}^{g}(x)=J_{\alpha}(x, T(x))\right\} .
$$

The family $\Omega^{\mathscr{f}}$ satisfies the assumption (A1) of Theorem 7; that is, $D_{\Omega^{\mathcal{f}}}=\bigcap_{\alpha \in \mathscr{A}} \operatorname{dom}\left(\omega_{\alpha}^{\mathscr{f}}\right) \neq \varnothing$.

Indeed, by (B1),

$$
\begin{aligned}
\forall_{x \in X} \quad\{\{y \in T(x): \\
\left.\left.\quad \forall_{\alpha \in \mathscr{A}}\left\{\omega_{\alpha}^{\mathscr{J}}(y) \leqslant \lambda_{\alpha} J_{\alpha}(x, y)\right\}\right\}=T(x)\right\} .
\end{aligned}
$$

Also, by Definition $1, \mathscr{J}=\left\{J_{\alpha}: X^{2} \rightarrow[0 ; \infty), \alpha \in \mathscr{A}\right\}$ and, by definition of $(X, T), \forall_{x \in X}\{\varnothing \neq T(x)\}$. Hence we conclude that $\forall_{x \in X}\left\{\varnothing \neq T(x) \subset D_{\Omega^{f}}\right\}$.

Step 2. The assumptions (A5) and (A6) of Theorem 7 hold where $\Upsilon=\left\{\varepsilon_{\alpha}=\gamma_{\alpha}^{0}-\lambda_{\alpha}, \alpha \in \mathscr{A}\right\}$ and $\Omega^{\mathscr{F}}$ is defined in Step 1.

Indeed, by assumption (V) (i.e., by assumption $\left.\forall_{x \in X_{\mathcal{F}}^{0}}\left\{\varnothing \neq Q_{\mathcal{F}, \Gamma^{0}-\Lambda ; T}(x) \in \mathrm{Cl}(X)\right\}\right)$ it follows that

$$
\begin{gathered}
\forall_{x \in X_{\mathcal{F}}^{0}} \quad\left\{\varnothing \neq Q_{\mathscr{f}, \Omega, Y ; T}(x)\right. \\
=\left\{y \in T(x) \cap X_{\mathscr{g}}^{0}:\right. \\
\forall_{\alpha \in \mathscr{A}}\left\{\omega_{\alpha}^{\mathcal{F}}(y)+\left(\gamma_{\alpha}^{0}-\lambda_{\alpha}\right) J_{\alpha}(x, y)\right. \\
\left.\left.\left.\leqslant \omega_{\alpha}^{\mathcal{F}}(x)\right\}\right\} \in \mathrm{Cl}(X)\right\} .
\end{gathered}
$$

Step 3. There exists $w \in X_{g}^{0}$ such that $w \in T(w)$.

This is a consequence of (I)-(V), Steps 1 and 2, and Theorem 7 .

Step 4. We now observe that $\forall_{w \in \operatorname{End}(T)} \forall_{\alpha \in \mathscr{A}}\left\{J_{\alpha}(w, w)=0\right\}$.

Otherwise, $\exists_{w_{0} \in \operatorname{End}(T)} \exists_{\alpha_{0} \in \mathscr{A}}\left\{J_{\alpha_{0}}\left(w_{0}, w_{0}\right)>0\right\}$. Consequently, for each $v \in\{1,2\}$,

$$
\begin{aligned}
J_{\alpha_{0}}\left(w_{0}, w_{0}\right) & =H_{v ; \alpha_{0}}^{\mathscr{g}}\left(\left\{w_{0}\right\},\left\{w_{0}\right\}\right) \\
& =H_{v ; \alpha_{0}}^{\mathcal{g}}\left(T\left(w_{0}\right), T\left(w_{0}\right)\right) \leqslant \lambda_{\alpha_{0}} J_{\alpha_{0}}\left(w_{0}, w_{0}\right),
\end{aligned}
$$

which is absurd.

Step 5. The assertions hold.

This follows from assumptions of Theorem 17, Steps 1-4, definition of $X_{\mathscr{g}}^{0}$, and Theorem 7 .
As a corollary of the above Theorems 17, 20, and 21 we have the following new fixed point and endpoint theorem for set-valued contractions with respect to $\mathscr{D}$-families (of Nadletype) in uniform spaces $(X, \mathscr{D})$.

Theorem 22. Let $(X, \mathscr{D})$ be a Hausdorff sequentially complete uniform space and let $v \in\{1,2\}$. Suppose also the following.

(I) A set-valued dynamic system $(X, T)$ satisfies $T: X \rightarrow$ $\mathrm{Cl}(X)$.

(II) There exists a family $\Lambda=\left\{\lambda_{\alpha} \in[0 ; 1), \alpha \in \mathscr{A}\right\}$ such that $(X, T)$ is a $\left(\mathscr{H}_{v}^{\mathscr{D}}, \Lambda\right)$-contraction on $X$.

(III) For each family $\Gamma=\left\{\gamma_{\alpha} \in(0 ; 1), \alpha \in \mathscr{A}\right\}$ satisfying $\forall_{\alpha \in \mathscr{A}}\left\{\lambda_{\alpha}<\gamma_{\alpha}\right\}$ and for each $x \in X$, let the set $Q_{\mathscr{D}, \Gamma-\Lambda ; T}(x)$ be defined by

$$
\begin{gathered}
Q_{\mathscr{D}, \Gamma-\Lambda ; T}(x)=\{y \in T(x): \\
\forall_{\alpha \in \mathscr{A}}\left\{d_{\alpha}(y, T(y))+\left(\gamma_{\alpha}-\lambda_{\alpha}\right) d_{\alpha}(x, y)\right. \\
\left.\left.\leqslant d_{\alpha}(x, T(x))\right\}\right\} .
\end{gathered}
$$

(IV) There exists a family $\Gamma^{0}=\left\{\gamma_{\alpha}^{0} \in(0 ; 1), \alpha \in \mathscr{A}\right\}$ satisfying $\forall_{\alpha \in \mathscr{A}}\left\{\lambda_{\alpha}<\gamma_{\alpha}^{0}\right\}$ and such that $\forall_{x \in X}\left\{Q_{\mathscr{D}, \Gamma^{0}-\Lambda ; T}(x)\right.$ $\neq \varnothing\}$.

The following hold.

(F1) (Closedness property) For each $x \in X, Q_{\mathscr{D}, \Gamma^{0}-\Lambda ; T}(x)$ is a closed subset in $X$.

(F2) (Fixed point theorem) $\operatorname{Fix}(T) \neq \varnothing$.

(F3) (Endpoint theorem) If, for each $x \in X$, each dynamic process $\left(w_{m}: m \in\{0\} \cup \mathbb{N}\right)$ starting at $w_{0}=$ $x$ and satisfying $\forall_{m \in\{0\} \cup \mathbb{N}}\left\{w_{m+1} \in T\left(w_{m}\right)\right\}$ satisfies $\forall_{m \in\{0\} \cup \mathbb{N}}\left\{w_{m+1} \in Q_{\mathscr{D}, \Gamma^{0}-\Lambda ; T}\left(w_{m}\right)\right\}$, then End $(T) \neq$ $\varnothing$.

We now state consequences of the above in metric spaces.

Definition 23. Let $(X, d)$ be a complete metric space, let $\mathscr{J}=$ $\left\{J: X^{2} \rightarrow[0 ; \infty)\right\} \in \mathbb{J}_{(X, d)}$, and let $v \in\{1,2\}$.

(a) Let

$$
\forall_{u \in X} \forall_{V \in \operatorname{Cl}(X)} \quad\{J(u, V)=\inf \{J(u, z): z \in V\}\} .
$$

Define $H_{v}^{\mathscr{f}}: \mathrm{Cl}(X)^{2} \rightarrow[0 ; \infty]$ as follows:

$$
\begin{aligned}
\forall_{A, B \in \mathrm{Cl}(X)} & H_{v}^{\mathcal{J}}(A, B) \\
& = \begin{cases}\max \left\{\sup _{u \in A} J(u, B), \sup _{z \in B} J(z, A)\right\}, & \text { if } v=1, \\
\sup _{u \in A} J(u, B), & \text { if } v=2 .\end{cases}
\end{aligned}
$$

(b) Let a set-valued dynamic system $(X, T)$ satisfy $T$ : $X \rightarrow \mathrm{Cl}(X)$. If $(X, T)$ satisfies

$\exists_{\lambda \in[0 ; 1)} \forall_{x, y \in X} \quad\left\{H_{v}^{\mathcal{g}}(T(x), T(y)) \leqslant \lambda J(x, y)\right\}$, then we say that $(X, T)$ is a $\left(H_{v}^{\mathcal{F}}, \lambda\right)$-contraction on $X$. 
(c) Let $X_{\mathscr{J}}^{0}=\{x \in X: J(x, x)=0\} . \mathscr{J}$ is said to be admissible if $X_{f}^{0} \neq \varnothing$.

(d) We say that $\mathscr{J}$ is continuous in $X$ if, for each $x_{0} \in X$ and for each sequence $\left(x_{m}: m \in \mathbb{N}\right)$ in $X$ such that $\lim _{m \rightarrow \infty} d\left(x_{m}, x_{0}\right)=0$, we have

$$
\liminf _{m \rightarrow \infty} J\left(x_{m}, x_{0}\right)=\liminf _{m \rightarrow \infty} J\left(x_{0}, x_{m}\right)=0 .
$$

Remark 24. Let $\mathscr{D}=\left\{d: X^{2} \rightarrow[0 ; \infty)\right\}$. It is clear that $\mathscr{D}$ is $\mathscr{J}$-family; $\mathscr{D}$ is admissible; $\mathscr{D}$ is continuous; and $X_{\mathscr{D}}^{0}=X$.

As corollaries from Theorems 17, 20, and 21 and their proofs we get the following three theorems concerning contractions with respect to $\mathscr{J} \in \mathbb{J}_{(X, d)}$ (of Nadler-type) in metric spaces $(X, d)$.

Theorem 25. Let $(X, d)$ be a complete metric space, $\mathscr{J}=\{J$ : $\left.X^{2} \rightarrow[0 ; \infty)\right\} \in \mathbb{J}_{(X, d)}$ and $v \in\{1,2\}$. Suppose also the following.

(I) $\mathcal{F}$ is admissible.

(II) A set-valued dynamic system $(X, T)$ satisfies $T: X \rightarrow$ $\mathrm{Cl}(X)$.

(III) There exists $\lambda \in(0 ; 1)$ such that $(X, T)$ is a $\left(H_{v}^{\mathcal{g}}, \lambda\right)$ contraction on $X$.

(IV) For each $\gamma \in(0 ; 1)$ satisfying $\lambda<\gamma$ and for each $x \in X$ let the set $Q_{\mathcal{f}, \gamma-\lambda ; T}(x)$ be defined by

$$
\begin{aligned}
Q_{\mathcal{F}, \gamma-\lambda ; T}(x)=\{y & \in T(x) \cap X_{\mathscr{g}}^{0}: J(y, T(y)) \\
& +(\gamma-\lambda) J(x, y) \leqslant J(x, T(x))\} .
\end{aligned}
$$

The following hold.

(G1) If there exists $\gamma^{0} \in(0 ; 1)$ satisfying $\lambda<\gamma^{0}$ and such that

$$
\begin{aligned}
& \forall_{x \in X_{\mathcal{F}}^{0}} \quad\left\{\left\{y \in T(x) \cap X_{\mathcal{g}}^{0}:\right.\right. \\
&\left.\left.\left\{\gamma^{0} J(x, y) \leqslant J(x, T(x))\right\}\right\} \neq \varnothing\right\},
\end{aligned}
$$

then $\forall_{x \in X_{\mathcal{f}}^{0}}\left\{Q_{\mathcal{F}, \gamma^{0}-\lambda ; T}(x) \neq \varnothing\right\}$.

(G2) If there exists $\gamma^{0} \in(0 ; 1)$ satisfying $\lambda<\gamma^{0}$ and such that, for each $x \in X_{\mathcal{q}}^{0}$, the map

$$
J(\cdot, T(\cdot))+\left(\gamma^{0}-\lambda\right) J(x, \cdot): T(x) \cap X_{\mathscr{F}}^{0} \longrightarrow[0 ; \infty)
$$

is $\left(T(x) \cap X_{\mathcal{f}}^{0}, X\right)$-lsc, then, for each $x \in X_{\mathcal{F}}^{0}$, $Q_{\mathscr{J}, \gamma^{0}-\lambda ; T}(x)$ is a closed subset in $X$.

(G3) Let $\mathscr{J}$ be continuous in $X$. Then, for each $\gamma \in(0,1)$ satisfying $\lambda<\gamma$ and for each $x \in X_{\mathscr{F}}^{0}, Q_{\mathcal{F}, \gamma-\lambda ; T}(x)$ is a closed subset in $X$.

Theorem 26. Let $(X, d)$ be a complete metric space, $\mathscr{J}=\{J$ : $\left.X^{2} \rightarrow[0 ; \infty)\right\} \in \mathbb{J}_{(X, d)}$ and $v \in\{1,2\}$. Suppose also the following.
(I) $\mathcal{J}$ is admissible.

(II) A set-valued dynamic system $(X, T)$ satisfies $T: X \rightarrow$ $\mathrm{Cl}(X)$.

(III) There exists $\lambda \in(0 ; 1)$ such that $(X, T)$ is a $\left(H_{v}^{\mathcal{g}}, \lambda\right)$ contraction on $X$.

(IV) For each $\gamma \in(0 ; 1)$ satisfying $\lambda<\gamma$ and for each $x \in X$ let the set $Q_{\mathscr{f}, \gamma-\lambda ; T}(x)$ be defined by

$$
\begin{aligned}
Q_{\mathcal{F}, \gamma-\lambda ; T}(x)=\left\{y \in T(x) \cap X_{\mathcal{F}}^{0}: J(y, T(y))\right. \\
+(\gamma-\lambda) J(x, y) \leqslant J(x, T(x))\} .
\end{aligned}
$$

(V) There exists $\gamma^{0} \in(0 ; 1)$ satisfying $\lambda<\gamma^{0}$ such that, for each $x \in X_{\mathscr{f}}^{0}, Q_{\mathscr{F}, \gamma^{0}-\lambda ; T}(x)$ is a nonempty closed subset in $X$.

The following hold.

(K1) (Fixed point theorem) $\operatorname{Fix}(T) \neq \varnothing$ and there exists $w \in \operatorname{Fix}(T)$ such that $J(w, w)=0$.

(K2) (Endpoint theorem) If, for each $x \in X_{g}^{0}$, each dynamic process $\left(w_{m}: m \in\{0\} \cup \mathbb{N}\right)$ starting at $w_{0}=$ $x$ and satisfying $\forall_{m \in\{0\} \cup \mathbb{N}}\left\{w_{m+1} \in T\left(w_{m}\right)\right\}$ satisfies $\forall_{m \in\{0\} \cup \mathbb{N}}\left\{w_{m+1} \in Q_{\mathcal{F}, \gamma^{0}-\lambda ; T}\left(w_{m}\right)\right\}$, then End $(T) \neq \varnothing$ and $\forall_{w \in \text { End (T) }}\{J(w, w)=0\}$.

Theorem 27. Let $(X, d)$ be a complete metric space, $\mathscr{D}=\{d$ : $\left.X^{2} \rightarrow[0 ; \infty)\right\}$ and $v \in\{1,2\}$. Suppose also the following.

(I) A set-valued dynamic system $(X, T)$ satisfies $T: X \rightarrow$ $\mathrm{Cl}(X)$.

(II) There exists $\lambda \in(0 ; 1)$ such that $(X, T)$ is a $\left(H_{v}^{D}, \lambda\right)$ contraction on $X$.

(III) For each $\gamma \in(0 ; 1)$ satisfying $\lambda<\gamma$ and for each $x \in X$ let the set $Q_{\mathscr{D}, \gamma-\lambda ; T}(x)$ be defined by

$$
\begin{aligned}
Q_{\mathscr{D}, \gamma-\lambda ; T}(x)=\{y & \in T(x): d(y, T(y)) \\
& +(\gamma-\lambda) d(x, y) \leqslant d(x, T(x))\} .
\end{aligned}
$$

The following hold.

(L1) (Nonemptness and closedness property) For each $\gamma \in$ $(0 ; 1)$ satisfying $\lambda<\gamma$ and for each $x \in X, Q_{\mathscr{D}, \gamma-\lambda ; T}(x)$ is a nonempty closed subset in $X$.

(L2) (Fixed point theorem) $\operatorname{Fix}(T) \neq \varnothing$.

(L3) (Endpoint theorem) If there exists $\gamma^{0} \in(0 ; 1)$ satisfying $\lambda<\gamma^{0}$ and such that, for each $x \in X$, each dynamic process $\left(w_{m}: m \in\{0\} \cup \mathbb{N}\right)$ starting at $w_{0}=$ $x$ and satisfying $\forall_{m \in\{0\} \cup \mathbb{N}}\left\{w_{m+1} \in T\left(w_{m}\right)\right\}$ satisfies $\forall_{m \in\{0\} \cup \mathbb{N}}\left\{w_{m+1} \in Q_{\mathscr{D}, \gamma^{0}-\lambda ; T}\left(w_{m}\right)\right\}$, then End $(T) \neq \varnothing$.

Remark 28. Theorem 27(L2) generalizes Theorem 12 (see Examples 5 and 6). 


\section{Fixed Point Theorems for Single-Valued Contractions (of Banach-Type) in Uniform and Metric Spaces}

Definition 29. Let $(X, \mathscr{D})$ be a Hausdorff sequentially complete uniform space, assume that $\mathscr{J}=\left\{J_{\alpha}: X^{2} \rightarrow[0 ; \infty)\right.$, $\alpha \in \mathscr{A}\} \in \mathbb{J}_{(X, \mathscr{D})}$ and let $v \in\{1,2\}$.

(a) Define on $X$ the distance $\mathscr{B}_{v}^{\mathscr{F}}, \mathscr{B}_{v}^{\mathscr{F}}=\left\{B_{v ; \alpha}^{\mathscr{F}}: X^{2} \rightarrow\right.$ $[0 ; \infty], \alpha \in \mathscr{A}\}$, as follows:

$$
\begin{aligned}
\forall_{\alpha \in \mathscr{A}} \forall_{x, y \in X} \quad B_{v, \alpha}^{\mathscr{g}}(x, y) & \\
\quad & = \begin{cases}\max \left\{J_{\alpha}(x, y), J_{\alpha}(y, x)\right\}, & \text { if } v=1, \\
J_{\alpha}(x, y), & \text { if } v=2 .\end{cases}
\end{aligned}
$$

(b) Let $(X, T)$ be a single-valued dynamic system, $T$ : $X \rightarrow X$. If $(X, T)$ satisfies

$$
\forall_{\alpha \in \mathscr{A}} \exists_{\lambda_{\alpha} \in[0 ; 1)} \forall_{x, y \in X} \quad\left\{B_{v, \alpha}^{\mathscr{g}}(T(x), T(y)) \leqslant \lambda_{\alpha} J_{\alpha}(x, y)\right\},
$$

then we say that $(X, T)$ is a $\left(\mathscr{B}_{v}^{\mathcal{F}}, \Lambda\right)$-contraction on $X$ for $\Lambda=\left\{\lambda_{\alpha}, \alpha \in \mathscr{A}\right\}$.

Remark 30. Each $\left(\mathscr{B}_{1}^{\mathcal{I}}, \Lambda\right)$-contraction on $X$ is $\left(\mathscr{B}_{2}^{\mathscr{f}}, \Lambda\right)$ contraction on $X$ but converse does not hold.

We use notations above and Theorem 21 in proving the following new fixed point theorem for single-valued contractions with respect to $\mathscr{J} \in \mathbb{I}_{(X, \mathscr{D})}$ (of Banach-type) in uniform spaces $(X, \mathscr{D})$.

Theorem 31. Let $(X, \mathscr{D})$ be a Hausdorf sequentially complete uniform space, let $\mathscr{J}=\left\{J_{\alpha}: X^{2} \rightarrow[0 ; \infty), \alpha \in \mathscr{A}\right\} \in \mathbb{J}_{(X, \mathscr{D})}$, and let $v \in\{1,2\}$. Suppose also the following.

(I) $\mathcal{J}$ is admissible.

(II) $(X, T)$ is a single-valued dynamic system, $T: X \rightarrow X$.

(III) There exists a family $\Lambda=\left\{\lambda_{\alpha} \in[0 ; 1), \alpha \in \mathscr{A}\right\}$ such that $(X, T)$ is a $\left(\mathscr{B}_{v}^{\mathcal{F}}, \Lambda\right)$-contraction on $X$ for $\Lambda$.

(IV) $T\left(X_{\mathscr{f}}^{0}\right) \subset X_{\mathscr{g}}^{0}$.

The following hold.

(M1) $T$ has a unique fixed point $w$ in $X$; that is, $T(w)=w$ and $\operatorname{Fix}(T)=\{w\}$.

(M2) $\forall_{\alpha \in \mathscr{A}}\left\{J_{\alpha}(w, w)=0\right\}$.

(M3) For each $w_{0} \in X$, the sequence $\left(w_{m}=T^{[m]}\left(w_{0}\right): m \in\right.$ $\mathbb{N})$ satisfies

$$
\begin{gathered}
\forall_{\alpha \in \mathscr{A}} \quad\left\{\lim _{m \rightarrow \infty} J_{\alpha}\left(w, w_{m}\right)=\lim _{m \rightarrow \infty} J_{\alpha}\left(w_{m}, w\right)=0\right\}, \\
\forall_{\alpha \in \mathscr{A}} \quad\left\{\lim _{m \rightarrow \infty} d_{\alpha}\left(w, w_{m}\right)=0\right\} .
\end{gathered}
$$

Proofs of (M1) and (M2). By Remark 30, Definition 29, and the assumptions (I)-(IV) of Theorem 31, we see that

$$
\begin{gathered}
\forall_{x \in X_{\mathscr{f}}^{0}} \forall_{\alpha \in \mathscr{A}} \quad\left\{J_{\alpha}\left(T(x), T^{[2]}(x)\right) \leqslant \lambda_{\alpha} J_{\alpha}(x, T(x))\right\}, \\
\forall_{x \in X_{\mathcal{F}}^{0}} \quad\left\{y=T(x) \in X_{\mathscr{f}}^{0}\right\} .
\end{gathered}
$$

Let now $\Delta=\left\{\delta_{\alpha} \in(0 ; 1), \alpha \in \mathscr{A}\right\}$ satisfying $\forall_{\alpha \in \mathscr{A}}\left\{\lambda_{\alpha}<\delta_{\alpha}\right\}$ be arbitrary and fixed. One then immediately finds that

$$
\begin{gathered}
\forall_{x \in X_{\mathcal{f}}^{0}} \forall_{\alpha \in \mathscr{A}} \quad\left\{J_{\alpha}\left(T(x), T^{[2]}(x)\right) \leqslant \lambda_{\alpha} J_{\alpha}(x, T(x))\right. \\
\left.+\left(1-\delta_{\alpha}\right) J_{\alpha}(x, T(x))\right\}
\end{gathered}
$$

or, equivalently,

$$
\begin{gathered}
\forall_{x \in X_{\mathscr{f}}^{0}} \forall_{\alpha \in \mathscr{A}} \quad\left\{J_{\alpha}(y, T(y))+\left(\delta_{\alpha}-\lambda_{\alpha}\right) J_{\alpha}(x, y)\right. \\
\left.\leqslant J_{\alpha}(x, T(x))\right\}
\end{gathered}
$$

where $y=T(x) \in X_{\mathscr{g}}^{0}$. Consequently, for each $x \in X_{\mathscr{g}}^{0}$, the singleton set

$$
\begin{aligned}
& Q_{\mathscr{g}, \Delta-\Lambda ; T}(x)=\{y=T(x) \in X_{\mathcal{F}}^{0}: \\
& \forall_{\alpha \in \mathscr{A}}\left\{J_{\alpha}(y, T(y))\right. \\
& \quad+\left(\delta_{\alpha}-\lambda_{\alpha}\right) J_{\alpha}(x, y) \\
&\left.\left.\leqslant J_{\alpha}(x, T(x))\right\}\right\} \\
&=\{T(x)\}
\end{aligned}
$$

is a nonempty closed subset in $X$.

From the above and Theorem 21 it follows that $T$ has a fixed point $w$ in $X$ (i.e., $w=T(w))$ and $\forall_{\alpha \in \mathscr{A}}\left\{J_{\alpha}(w, w)=0\right\}$ ).

It remains to verify that $\operatorname{Fix}(T)=\{w\}$. Suppose that $\{u, w\} \subset \operatorname{Fix}(T)$. By Definition 29 and assumptions of Theorem 31, we obtain that, if $v=1$, then

$$
\begin{aligned}
& \forall_{\alpha \in \mathscr{A}} \exists_{\lambda_{\alpha} \in[0 ; 1)} \\
& \left\{\left[J_{\alpha}(u, w) \leqslant \max \left\{J_{\alpha}(u, w), J_{\alpha}(w, u)\right\}\right.\right. \\
& =\max \left\{J_{\alpha}(T(u), T(w)),\right. \\
& \left.\left.J_{\alpha}(T(w), T(u))\right\} \leqslant \lambda_{\alpha} J_{\alpha}(u, w)\right] \\
& \wedge\left[J_{\alpha}(w, u) \leqslant \max \left\{J_{\alpha}(u, w), J_{\alpha}(w, u)\right\}\right. \\
& =\max \left\{J_{\alpha}(T(u), T(w)),\right. \\
& \left.\left.\left.\quad J_{\alpha}(T(w), T(u))\right\} \leqslant \lambda_{\alpha} J_{\alpha}(w, u)\right]\right\}
\end{aligned}
$$

and, if $v=2$, then

$$
\begin{aligned}
& \forall_{\alpha \in \mathscr{A}} \exists_{\lambda_{\alpha} \in[0 ; 1)} \\
& \left\{\left[J_{\alpha}(u, w)=J_{\alpha}(T(u), T(w)) \leqslant \lambda_{\alpha} J_{\alpha}(u, w)\right]\right. \\
& \wedge\left[J_{\alpha}(w, u)=J_{\alpha}(T(w), T(u))\right. \\
& \left.\left.\leqslant \lambda_{\alpha} J_{\alpha}(w, u)\right]\right\} .
\end{aligned}
$$


Hence $\forall_{\alpha \in \mathscr{A}}\left\{J_{\alpha}(u, w)=J_{\alpha}(w, u)=0\right\}$. From this information, by Remark 3(b), we deduce that $u=w$.

Therefore, the assertions (M1) and (M2) hold.

Proof of (M3). Let now $w_{0} \in X$ be arbitrary and fixed and put $\left(w_{m}=T^{[m]}\left(w_{0}\right): m \in \mathbb{N}\right)$. By Definition 29, assumptions of Theorem 31 and the fact that $T^{[m]}(w)=w$ for $m \in \mathbb{N}$, we obtain that, if $v=1$, then

$$
\begin{aligned}
\forall_{\alpha \in \mathscr{A}} \exists_{\lambda_{\alpha} \in[0 ; 1)} \forall_{m \in \mathbb{N}} \quad\{[ & J_{\alpha}\left(w, w_{m+1}\right) \\
& \leqslant \max \left\{J_{\alpha}\left(w_{m+1}, w\right), J_{\alpha}\left(w, w_{m+1}\right)\right\} \\
& \left.\leqslant \lambda_{\alpha} J_{\alpha}\left(w, w_{m}\right)\right] \\
& \wedge\left[J_{\alpha}\left(w_{m+1}, w\right)\right. \\
& \leqslant \max \left\{J_{\alpha}\left(w_{m+1}, w\right), J_{\alpha}\left(w, w_{m+1}\right)\right\} \\
& \left.\left.\leqslant \lambda_{\alpha} J_{\alpha}\left(w_{m}, w\right)\right]\right\},
\end{aligned}
$$

and, if $v=2$, then

$$
\begin{aligned}
\forall_{\alpha \in \mathscr{A}} \exists_{\lambda_{\alpha} \in[0 ; 1)} \forall_{m \in \mathbb{N}} \quad & \left\{\left[J_{\alpha}\left(w, w_{m+1}\right)\right.\right. \\
& \left.\leqslant \lambda_{\alpha} J_{\alpha}\left(w, w_{m}\right)\right] \\
& \left.\wedge\left[J_{\alpha}\left(w_{m+1}, w\right) \leqslant \lambda_{\alpha} J_{\alpha}\left(w_{m}, w\right)\right]\right\} .
\end{aligned}
$$

Hence

$$
\begin{aligned}
\forall_{\alpha \in \mathscr{A}} \exists_{\lambda_{\alpha} \in[0 ; 1)} \forall_{m \in \mathbb{N}} & \left\{\left[J_{\alpha}\left(w, w_{m+1}\right)\right.\right. \\
& \left.\leqslant \lambda_{\alpha}^{m} J_{\alpha}\left(w, w_{0}\right)\right] \\
& \wedge\left[J_{\alpha}\left(w_{m+1}, w\right)\right. \\
& \left.\left.\leqslant \lambda_{\alpha}^{m} J_{\alpha}\left(w_{0}, w\right)\right]\right\} .
\end{aligned}
$$

This gives the assertion (72), since, by Definition 1,

$$
\forall_{\alpha \in \mathscr{A}} \quad\left\{\left[J_{\alpha}\left(w, w_{0}\right)<+\infty\right],\left[J_{\alpha}\left(w_{0}, w\right)<+\infty\right]\right\} .
$$

Finally, let $w_{0} \in X$ be arbitrary and fixed and put $\left(w_{m}=\right.$ $\left.T^{[m]}\left(w_{0}\right): m \in \mathbb{N}\right),\left(x_{m}=w: m \in \mathbb{N}\right)$, and $\left(y_{m}=w_{m}: m \in\right.$ $\mathbb{N})$. Using assertion (M2), we then have

$$
\forall_{\alpha \in \mathscr{A}} \quad\left\{\lim _{n \rightarrow \infty} \sup _{m>n} J_{\alpha}\left(x_{n}, x_{m}\right)=0\right\},
$$

and, using assertion (72), we get

$$
\forall_{\alpha \in \mathscr{A}} \quad\left\{\lim _{m \rightarrow \infty} J_{\alpha}\left(x_{m}, y_{m}\right)=0\right\} .
$$

Hence, using Definition $1(\mathscr{J} 2)$, we find

$$
\forall_{\alpha \in \mathscr{A}} \quad\left\{\lim _{m \rightarrow \infty} d_{\alpha}\left(x_{m}, y_{m}\right)=\lim _{m \rightarrow \infty} d_{\alpha}\left(w, w_{m}\right)=0\right\} .
$$

Thus (73) holds.
Remark 32. (a) Theorem 31 includes Theorem 11 [16] and the result of [52]. Theorem 31 is different from Theorem 11 [16] and the result of [52] even in metric spaces and in uniform spaces, respectively (see Examples 4 and 7).

(b) Let $v \in\{1,2\}$. Assumptions (III) and (IV) imply that $\left(X_{\mathscr{f}}^{0}, T\right)$ is also a $\left(\mathscr{B}_{v}^{\mathscr{f}}, \Lambda\right)$-contraction on $X_{\mathscr{f}}^{0}$. However, the dynamic systems $(X, T)$ and $\left(X_{\mathscr{g}}^{0}, T\right)$ are not necessarily $\left(\mathscr{B}_{v}^{\mathscr{D}}, \Lambda\right)$-contractions on $X$ or $X_{\mathscr{f}}^{0}$, respectively (see Examples 4 and 7).

(c) Assumptions (II) and (IV) and assertions (M1) and (M2) imply that $w \in X_{\mathcal{F}}^{0}$ is a unique fixed point of $(X, T)$ and $\left(X_{\mathscr{g}}^{0}, T\right)$. Assertion (M3) implies, in particular, that, for each starting point $w_{0}$ of the space $X$, the dynamic process of the system $(X, T)$ converges to $w$.

The above has interesting implications for metric spaces.

Definition 33. Let $(X, \mathscr{D})$ be a complete metric space, assume that $\mathscr{J}=\left\{J: X^{2} \rightarrow[0 ; \infty)\right\} \in \mathbb{J}_{(X, d)}$ and let $v \in\{1,2\}$.

(a) Define on $X$ the distance $B_{v}^{\mathcal{F}}: X^{2} \rightarrow[0, \infty]$ as follows:

$$
\begin{aligned}
\forall_{x, y \in X} \quad B_{i}^{\mathcal{F}}(x, y) & \\
& = \begin{cases}\max \{J(x, y), J(y, x)\}, & \text { if } v=1, \\
J(x, y), & \text { if } v=2 .\end{cases}
\end{aligned}
$$

(b) Let $(X, T)$ be a single-valued dynamic system, $T$ : $X \rightarrow X$. If $(X, T)$ satisfies

$$
\exists_{\lambda \in[0 ; 1)} \forall_{x, y \in X} \quad\left\{B_{v}^{\mathcal{F}}(T(x), T(y)) \leqslant \lambda J(x, y)\right\}
$$

then we say that $(X, T)$ is a $\left(B_{v}^{\mathcal{g}}, \lambda\right)$-contraction on $X$ for $\lambda$.

As a corollary from Theorem 31 and its proof we get the following fixed point theorem for single-valued contractions with respect to $\mathscr{J} \in \mathbb{J}_{(X, d)}$ (of Banach-type) in metric spaces $(X, d)$.

Theorem 34. Let $(X, d)$ be a complete metric space, $\mathscr{J}=\{J$ : $\left.X^{2} \rightarrow[0 ; \infty)\right\} \in \mathbb{J}_{(X, d)}$ and $v \in\{1,2\}$. Suppose also the following.

(I) $\mathcal{J}$ is admissible.

(II) $(X, T)$ is a single-valued dynamic system, $T: X \rightarrow X$.

(III) There exists $\lambda \in[0 ; 1)$ such that $(X, T)$ is a $\left(B_{v}^{\mathcal{g}}, \lambda\right)$ contraction on $X$ for $\lambda$.

(IV) $T\left(X_{\mathcal{g}}^{0}\right) \subset X_{\mathcal{g}}^{0}$.

The following hold.

(S1) $T$ has a unique fixed point $w$ in $X$ (i.e., $T(w)=w$ and $\operatorname{Fix}(T)=\{w\})$

(S2) $J(w, w)=0$.

(S3) For each $w_{0} \in X$, the sequence $\left(w_{m}=T^{[m]}\left(w_{0}\right): m \in\right.$ $\mathbb{N}$ ) satisfies $\lim _{m \rightarrow \infty} J\left(w, w_{m}\right)=\lim _{m \rightarrow \infty} J\left(w_{m}, w\right)=$ 0 and $\lim _{m \rightarrow \infty} d\left(w, w_{m}\right)=0$. 
Remark 35. Theorem 34 generalizes Theorem 11 (see Example 7).

\section{Examples Illustrating the Results}

The following example describes some $\mathscr{J}$-family in metric spaces.

Example 1. Let $(X, d)$ be a metric space. Let the set $E \subset X$, containing at least two different points, be arbitrary and fixed and let $c>0$ satisfy $\delta(E)<c$ where $\delta(E)=\sup \{d(x, y)$ : $x, y \in E\}$. Let $J: X^{2} \rightarrow[0 ; \infty)$ be defined by the formulae:

$$
J(x, y)= \begin{cases}d(x, y), & \text { if } E \cap\{x, y\}=\{x, y\}, \\ c, & \text { if } E \cap\{x, y\} \neq\{x, y\},\end{cases}
$$

$x, y \in X$. Then $\mathscr{J}=\left\{J: X^{2} \rightarrow[0 ; \infty)\right\} \in \mathbb{J}_{(X, d)}$ (see $[6$, Example 6.12]).

The following example illustrates the Theorem 26(K1) in the case when $\mathscr{J}=\left\{J: X^{2} \rightarrow[0 ; \infty)\right\} \in \mathbb{J}_{(X, d)}, J \neq d$.

Example 2. Let $X=[0 ; 6]$ be a complete metric space with a metric $d: X^{2} \rightarrow[0 ; \infty), d(x, y)=|x-y|, x, y \in X$. Let $T_{1}: X \rightarrow \mathrm{Cl}(X)$ be of the form:

$$
T_{1}(x)= \begin{cases}{[1 ; 2],} & \text { if } x \in[0 ; 6), \\ {[4 ; 5],} & \text { if } x=6\end{cases}
$$

Let $E=[0 ; 3) \cup(3 ; 6)$ and let $J$ be of the form:

$$
J(x, y)= \begin{cases}d(x, y) & \text { if } E \cap\{x, y\}=\{x, y\} \\ 8 & \text { if } E \cap\{x, y\} \neq\{x, y\} .\end{cases}
$$

Clearly, $\mathscr{J} \in \mathbb{J}_{(X, d)}$ (Example 1).

We observe that $X_{\mathscr{f}}^{0}=[0 ; 3) \cup(3 ; 6) \neq \emptyset$.

Let $\lambda=3 / 4$. We show that $\left(X, T_{1}\right)$ is a $\left(H_{1}^{\mathscr{g}}, 3 / 4\right)$ contraction on $X$. Indeed, let $x, y \in X$ be arbitrary and fixed. We consider three cases.

Case 1. If $x, y \in[0 ; 6)$, then we have that $T_{1}(x)=T_{1}(y)=$ $[1 ; 2]$ and $H_{1}^{\mathcal{F}}\left(T_{1}(x), T_{1}(y)\right)=0 \leqslant(3 / 4) J(x, y)=\lambda J(x, y)$.

Case 2. If $x \in[0 ; 6)$ and $y=6$, then $y \notin E, J(x, y)=8$, $T_{1}(x)=[1 ; 2]$ and $T_{1}(y)=[4 ; 5]$. Hence, we calculate the following.

(2.1) For $u \in T_{1}(x), J\left(u, T_{1}(y)\right)=d(u,[4 ; 5])=4-u$ and, consequently, $\sup \left\{J\left(u, T_{1}(y)\right): u \in T_{1}(x)\right\}=3$.

(2.2) For $v \in T_{1}(y), J\left(v, T_{1}(x)\right)=d(v,[1 ; 2])=v-2$ and, consequently, $\sup \left\{J\left(v, T_{1}(x)\right): v \in T_{1}(y)\right\}=3$.

(2.3) By (2.1) and (2.2), for $x \in[0 ; 6)$ and $y=6$,

$$
\begin{aligned}
H_{1}^{g} & \left(T_{1}(x), T_{1}(y)\right) \\
= & \max \left\{\sup \left\{J\left(u, T_{1}(y)\right): u \in T_{1}(x)\right\},\right. \\
& \left.\sup \left\{J\left(v, T_{1}(x)\right): v \in T_{1}(y)\right\}\right\} \\
= & 3 \leqslant 6=\lambda \cdot 8=\lambda J(x, y) .
\end{aligned}
$$

Case 3. If $x=6$ and $y \in X \backslash\{6\}$, then also (92) holds.

By Cases $1-3,\left(X, T_{1}\right)$ is a $\left(H_{1}^{\mathscr{g}}, \lambda\right)$-contraction on $X$.

Now, let $\gamma^{0}=7 / 8$. We prove that, for each $x \in X_{g}^{0}$, $Q_{\mathscr{F}, \gamma^{0}-\lambda ; T_{1}}(x)$ is a nonempty closed subset in $X$. Indeed, for each $x \in X_{\mathscr{g}}^{0}=[0 ; 3) \cup(3 ; 6)$, we have $T_{1}(x)=[1 ; 2] \subset X_{\mathscr{g}}^{0}$, $\forall_{y \in T_{1}(x)=[1 ; 2]}\left\{J\left(y, T_{1}(y)\right)=0\right\}$ and

$$
\begin{aligned}
& Q_{\mathscr{F}, \gamma^{0}-\lambda ; T_{1}}(x) \\
& \quad=\left\{y \in[1 ; 2]: J\left(y, T_{1}(y)\right)+\left(\frac{1}{8}\right) J(x, y) \leqslant J\left(x, T_{1}(x)\right)\right\} \\
& \quad=\left\{y \in[1 ; 2]:\left(\frac{1}{8}\right) d(x, y) \leqslant d(x,[1 ; 2])\right\} \\
& \quad=\left\{y \in[1 ; 2]:\left(\frac{1}{8}\right)|y-x| \leqslant d(x,[1 ; 2])\right\} .
\end{aligned}
$$

This implies the following.

Case 1. If $x \in[0 ; 1]$, then

$$
\begin{aligned}
Q_{\mathscr{f}, \gamma^{0}-\lambda ; T_{1}}(x) & =\{y \in[1 ; 2]: y-x \leqslant 8 d(x,[1 ; 2])\} \\
& =\{y \in[1 ; 2]: y \leqslant 8-7 x\} .
\end{aligned}
$$

Case 2. If $x \in(1 ; 2]$, then

$$
\begin{aligned}
Q_{\mathscr{F}, \gamma^{0}-\lambda ; T_{1}}(x) & =\left\{y \in[1 ; 2]:\left(\frac{1}{8}\right)|y-x| \leqslant d(x,[1 ; 2])=0\right\} \\
& =\{x\} .
\end{aligned}
$$

Case 3. If $x \in(2 ; 3) \cup(3 ; 6)$, then

$$
\begin{aligned}
Q_{\mathscr{F}, \gamma^{0}-\lambda ; T_{1}}(x) & =\{y \in[1 ; 2]: x-y \leqslant 8(x-2)\} \\
& =\{y \in[1 ; 2]: y \geqslant 16-7 x\} .
\end{aligned}
$$

Assumptions of Theorem 26(K1) hold for $v=1, \operatorname{Fix}\left(T_{1}\right)=$ $[1 ; 2]$, and, for each $w \in \operatorname{Fix}\left(T_{1}\right), J(w, w)=0$.

The following example illustrates the Theorem 26(K2) in the case when $\mathscr{J}=\{J\}, J \neq d$.

Example 3. Let $X, E, \mathcal{J}, \lambda=3 / 4$, and $\gamma^{0}=7 / 8$ be such as in Example 2 and let $T_{2}: X \rightarrow \mathrm{Cl}(X)$ be of the form:

$$
T_{2}(x)= \begin{cases}\{1\} & \text { for } x \in[0 ; 2) \cup\{3\} \cup[5 ; 6) \\ \{2\} & \text { for } x \in[2 ; 3) \cup(3 ; 5) \\ {[4 ; 5]} & \text { for } x=6\end{cases}
$$

Then $X_{g}^{0}=[0 ; 3) \cup(3 ; 6) \neq \emptyset$ and, by analogous considerations as in Example 2, we obtain that $\left(X, T_{2}\right)$ is a $\left(H_{1}^{\mathcal{F}}, 3 / 4\right)$-contraction on $X$. 
Next, let us observe that, for $x \in X_{\mathscr{g}}^{0}$,

$$
\begin{aligned}
Q_{\mathcal{F}, \gamma^{0}-\lambda ; T_{2}}(x) & \\
=\{y & \in T_{2}(x): J\left(y, T_{2}(y)\right) \\
& \left.\quad+\left(\frac{1}{8}\right) J(x, y) \leqslant J\left(x, T_{2}(x)\right)\right\} .
\end{aligned}
$$

Hence we have the following.

Case 1. If $x \in[0 ; 2) \cup\{3\} \cup[5 ; 6)$, then $T_{2}(x)=\{1\}$, $J\left(y, T_{2}(y)\right)=J(1,1)=0$ for $y \in T_{2}(x)$ and, consequently,

$$
\begin{aligned}
Q_{\mathscr{g}, \gamma^{0}-\lambda ; T_{2}}(x) & =\left\{y \in\{1\}: J(1,1)+\left(\frac{1}{8}\right) J(x, 1) \leqslant J(x, 1)\right\} \\
& =\left\{y \in\{1\}:\left(\frac{1}{8}\right)|x-1| \leqslant|x-1|\right\}=\{1\} .
\end{aligned}
$$

Case 2. If $x \in[2 ; 3) \cup(3 ; 5)$, then $T_{2}(x)=\{2\}, J\left(y, T_{2}(y)\right)=$ $J(2,2)=0$ for $y \in T_{2}(x)$ and, consequently,

$$
\begin{aligned}
Q_{\mathscr{g}, \gamma^{0}-\lambda ; T_{2}}(x) & =\left\{y \in\{2\}: J(2,2)+\left(\frac{1}{8}\right) J(x, 2) \leqslant J(x, 2)\right\} \\
& =\left\{y \in\{2\}:\left(\frac{1}{8}\right)|x-2| \leqslant|x-2|\right\}=\{2\} .
\end{aligned}
$$

Therefore, for each $x \in X_{\mathscr{g}}^{0}$, each dynamic process $\left(w_{m}: m \in\right.$ $\{0\} \cup \mathbb{N}$ ) starting at $w_{0}=x$ and satisfying $\forall_{m \in\{0\} \cup \mathbb{N}}\left\{w_{m+1} \in\right.$ $\left.T\left(w_{m}\right)\right\}$ satisfies $\forall_{m \in\{0\} \cup \mathbb{N}}\left\{w_{m+1} \in Q_{\mathcal{F}, \gamma^{0}-\lambda ; T}\left(w_{m}\right)\right\}$.

Assumptions of Theorem 26(K2) hold, $\operatorname{End}\left(T_{2}\right)=\{1,2\}$ and $J(1,1)=J(2,2)=0$.

The following example illustrates the Theorem 34 in the case when $\mathscr{J}=\left\{J: X^{2} \rightarrow[0 ; \infty)\right\} \in \mathbb{J}_{(X, d)}, J \neq d$.

Example 4. Let $X=\{1,2,3,4\} \cup[5 ; 6]$ be a metric space with a metric $d: X^{2} \rightarrow[0 ; \infty), d(x, y)=|x-y|, x, y \in X$. Let $E=\{1,2,4,5\}$ and let $J$ be of the form:

$$
J(x, y)= \begin{cases}d(x, y), & \text { if } E \cap\{x, y\}=\{x, y\}, \\ 8, & \text { if } E \cap\{x, y\} \neq\{x, y\} .\end{cases}
$$

Clearly, $\mathscr{J}=\left\{J: X^{2} \rightarrow[0 ; \infty)\right\}$ is a $\mathscr{J}$-family on $X$ (Example 1).

Let $\lambda=3 / 4$ and let $T_{3}: X \rightarrow X$ be of the form:

$$
T_{3}(x)= \begin{cases}2, & \text { for } x \in\{1,2,3\} \cup(5 ; 6], \\ 1, & \text { for } x \in\{4,5\} .\end{cases}
$$

Then $X_{\mathscr{g}}^{0}=\{1,2,4,5\} \neq \emptyset$ and $T_{3}: X_{\mathscr{g}}^{0} \rightarrow X_{g}^{0}$. Thus assumption (IV) of Theorem 34 holds.

We see that

$$
\begin{gathered}
\forall_{x, y \in X} \quad\left\{\max \left\{J\left(T_{3}(x), T_{3}(y)\right), J\left(T_{3}(y), T_{3}(x)\right)\right\}\right. \\
=J\left(T_{3}(x), T_{3}(y)\right) \\
\left.=d\left(T_{3}(x), T_{3}(y)\right) \leqslant \lambda J(x, y)\right\} ;
\end{gathered}
$$

that is, $\left(X, T_{3}\right)$ is a $\left(B_{1}^{\mathcal{g}}, \lambda\right)$-contraction on $X$. Indeed, we have the following.

Case 1. If $x \in\{1,2,3\} \cup(5 ; 6]$ and $y \in\{4,5\}$, then

$$
\begin{gathered}
1=d(2,1)=J(2,1)=J\left(T_{3}(x), T_{3}(y)\right), \\
\lambda J(x, y)= \begin{cases}3 \lambda=\frac{9}{4} & \text { for } x=1, y=4 \text { or } \quad x=2, y=5, \\
2 \lambda=\frac{3}{2} & \text { for } x=2, y=4, \\
4 \lambda=3 & \text { for } x=1, y=5, \\
8 \lambda=6 & \text { for } x \in\{3\} \cup(5 ; 6], y \in\{4,5\} .\end{cases}
\end{gathered}
$$

Case 2. If $x, y \in\{1,2,3\} \cup(5 ; 6]$, then

$$
0=d(2,2)=J(2,2)=J\left(T_{3}(x), T_{3}(y)\right) .
$$

Case 3. If $x, y \in\{4,5\}$, then

$$
0=d(1,1)=J(1,1)=J\left(T_{3}(x), T_{3}(y)\right) .
$$

Assumptions of Theorem 34 hold and the assertions (S1)(S3) are as follows. $\operatorname{Fix}\left(T_{3}\right)=\{2\}, J(2,2)=0$ and, for each $w_{0} \in X$, the sequence $\left(w_{m}=T^{[m]}\left(w_{0}\right): m \in \mathbb{N}\right)$ satisfies

$$
\begin{gathered}
\forall_{w_{0} \in X} \quad\left\{\lim _{m \rightarrow \infty} J\left(2, w_{m}\right)=\lim _{m \rightarrow \infty} J\left(w_{m}, 2\right)\right. \\
\left.=\lim _{m \rightarrow \infty} d\left(w_{m}, 2\right)=0\right\} .
\end{gathered}
$$

\section{Comparisons of Our Results with Nadler's and Banach's Results}

It is worth noticing that our results in metric spaces include Nadler's and Banach's results. Clearly, it is not otherwise. More precisely we have the following.

(a) In Examples 5 and 6 below we show that, for each $\lambda \in[0 ; 1)$, the set-valued dynamic systems $\left(X, T_{1}\right)$ and $\left(X, T_{2}\right)$ defined in Examples 2 and 3, respectively, are $\operatorname{not}\left(H^{d}, \lambda\right)$-contractions on $X$ and thus we cannot use Theorem 12.

(b) In Example 7 we show that, for each $\lambda \in[0 ; 1)$, the single-valued dynamic system $\left(X, T_{3}\right)$ defined in Example 4 is not $(d, \lambda)$-contractions on $X$ and thus we cannot use Theorem 11 .

Therefore, in our concepts of $\left(\mathscr{H}_{v}^{\mathcal{g}}, \Lambda\right)$-contractive setvalued dynamic systems and $\left(\mathscr{B}_{v}^{\mathscr{J}}, \Lambda\right)$-contractive singlevalued dynamic systems, $v \in\{1,2\}$, the existence of $\mathscr{J}$-family such that $\mathscr{J} \neq \mathscr{D}$ is essential.

Example 5. Let $(X, d)$ and $T_{1}$ be such as in Example 2 and let $\mathscr{J}=\{d\}$. We observe that $X_{\mathcal{g}}^{0}=X$. 
Next, we see that, for each $\lambda \in[0 ; 1),\left(X, T_{1}\right)$ is not a $\left(H^{d}, \lambda\right)$-contraction on $X$. Indeed, suppose that

$$
\exists_{\lambda \in[0 ; 1)} \forall_{x, y \in X} \quad\left\{H^{d}\left(T_{1}(x), T_{1}(y)\right) \leqslant \lambda d(x, y)\right\} .
$$

Then, in particular, for $x_{0}=3 \in X$ and $y_{0}=6 \in X$, we obtain the following.

(1) $T_{1}\left(x_{0}\right)=[1 ; 2]$ and $T_{1}\left(y_{0}\right)=[4 ; 5]$.

(2) For $u \in T_{1}\left(x_{0}\right)=[1 ; 2], d\left(u, T_{1}\left(y_{0}\right)\right)=d(u,[4 ; 5])=$ $4-u$ and, consequently,

$$
\sup \left\{d\left(u, T_{1}\left(y_{0}\right)\right): u \in T_{1}\left(x_{0}\right)\right\}=3 .
$$

(3) For $z \in T_{1}\left(y_{0}\right)=[4 ; 5], d\left(z, T_{1}\left(x_{0}\right)\right)=d(z,[1 ; 2])=$ $z-2$ and, consequently,

$$
\sup \left\{d\left(z, T_{1}\left(x_{0}\right)\right): z \in T_{1}\left(y_{0}\right)\right\}=3 \text {. }
$$

(4) By (2) and (3),

$$
\begin{aligned}
& H^{d}\left(T_{1}\left(x_{0}\right), T_{1}\left(y_{0}\right)\right) \\
& \quad=\max \left\{\sup _{u \in T_{1}\left(x_{0}\right)} d\left(u, T_{1}\left(y_{0}\right)\right), \sup _{z \in T_{1}\left(y_{0}\right)} d\left(z, T_{1}\left(x_{0}\right)\right)\right\}=3 .
\end{aligned}
$$

Hence, we get

$$
\begin{gathered}
\forall_{\lambda \in[0 ; 1)}\left\{3=H^{d}\left(T_{1}\left(x_{0}\right), T_{1}\left(y_{0}\right)\right) \leqslant \lambda d\left(x_{0}, y_{0}\right)\right. \\
\left.<d\left(x_{0}, y_{0}\right)=d(3,6)=3\right\},
\end{gathered}
$$

which is absurd.

Example 6. Let $(X, d)$ and $T_{2}$ be such as in Example 3 and let $\mathcal{J}=\{d\}$. By similar argumentation as in Example 5, we observe that, for each $\lambda \in[0 ; 1),\left(X, T_{2}\right)$ is not a $\left(H^{d}, \lambda\right)$ contraction on $X$.

Example 7. Let $(X, d)$ and $T_{3}$ be such as in Example 4 and let $\mathscr{J}=\mathscr{D}=\{d\}$. Clearly, $X_{\mathscr{D}}^{0}=X$.

We observe that, for each $\lambda \in[0 ; 1),\left(X, T_{3}\right)$ is not a $(d, \lambda)$ contraction on $X$. Otherwise, by Definition 29 for $\mathcal{J}=\{d\}$ (or by (9)), the following holds:

$$
\exists_{\lambda \in[0 ; 1)} \forall_{x, y \in X} \quad\left\{d\left(T_{3}(x), T_{3}(y)\right) \leqslant \lambda d(x, y)\right\} .
$$

However, in particular, for $x_{0}=3 \in X$ and $y_{0}=4 \in X$, we get $d\left(x_{0}, y_{0}\right)=1$ and then

$$
\begin{gathered}
\forall_{\lambda \in[0 ; 1)} \quad\left\{1=d(2,1)=d\left(T_{3}\left(x_{0}\right), T_{3}\left(y_{0}\right)\right)\right. \\
\left.\leqslant \lambda d\left(x_{0}, y_{0}\right)=\lambda<1\right\}
\end{gathered}
$$

which is absurd. This gives that the condition (113) does not hold.

\section{Conflict of Interests}

The authors declare that there is no conflict of interests regarding the publication of this paper.

\section{References}

[1] D. Tataru, "Viscosity solutions of Hamilton-Jacobi equations with unbounded nonlinear terms," Journal of Mathematical Analysis and Applications, vol. 163, no. 2, pp. 345-392, 1992.

[2] O. Kada, T. Suzuki, and W. Takahashi, "Nonconvex minimization theorems and fixed point theorems in complete metric spaces," Mathematica Japonica, vol. 44, no. 2, pp. 381-391, 1996.

[3] T. Suzuki, "Generalized distance and existence theorems in complete metric spaces," Journal of Mathematical Analysis and Applications, vol. 253, no. 2, pp. 440-458, 2001.

[4] L.-J. Lin and W.-S. Du, "Ekeland's variational principle, minimax theorems and existence of nonconvex equilibria in complete metric spaces," Journal of Mathematical Analysis and Applications, vol. 323, no. 1, pp. 360-370, 2006.

[5] I. Vályi, "A general maximality principle and a fixed point theorem in uniform space," Periodica Mathematica Hungarica, vol. 16, no. 2, pp. 127-134, 1985.

[6] K. Włodarczyk and R. Plebaniak, "Maximality principle and general results of Ekeland and Caristi types without lower semicontinuity assumptions in cone uniform spaces with generalized pseudodistances," Fixed Point Theory and Applications, vol. 2010, Article ID 175453, 35 pages, 2010.

[7] K. Włodarczyk and R. Plebaniak, "A fixed point theorem of Subrahmanyam type in uniform spaces with generalized pseudodistances," Applied Mathematics Letters, vol. 24, no. 3, pp. 325-328, 2011.

[8] J.-P. Aubin and J. Siegel, "Fixed points and stationary points of dissipative multivalued maps," Proceedings of the American Mathematical Society, vol. 78, no. 3, pp. 391-398, 1980.

[9] J.-P. Aubin and I. Ekeland, Applied Nonlinear Analysis, John Wiley \& Sons, 1984.

[10] J.-P. Aubin and H. Frankowska, Set-Valued Analysis, Birkhäuser, Boston, Mass, USA, 1990.

[11] G. X.-Z. Yuan, KKM Theory and Applications in Nonlinear Analysis, Marcel Dekker, New York, NY, USA, 1999.

[12] J. Caristi, "Fixed point theorems for mappings satisfying inwardness conditions," Transactions of the American Mathematical Society, vol. 215, pp. 241-251, 1976.

[13] I. Ekeland, "Remarques sur les problémes variationnels, I," Comptes Rendus de l'Académie des Sciences (Paris)—Series A-B, vol. 275, pp. 1057-1059, 1972.

[14] I. Ekeland, "On the variational principle," Journal of Mathematical Analysis and Applications, vol. 47, no. 2, pp. 324-353, 1974.

[15] I. Ekeland, "Nonconvex minimization problems," Bulletin of the American Mathematical Society, vol. 1, no. 3, pp. 443-474, 1979.

[16] S. Banach, "Sur les opérations dans les ensembles abstraits et leur application aux équations intégrales," Fundamenta Mathematicae, vol. 3, no. 1, pp. 133-181, 1922.

[17] S. B. Nadler, "Multi-valued contraction mappings," Pacific Journal of Mathematics, vol. 30, pp. 475-488, 1969.

[18] A. Amini-Harandi, "Endpoints of set-valued contractions in metric spaces," Nonlinear Analysis: Theory, Methods \& Applications, vol. 72, no. 1, pp. 132-134, 2010.

[19] H. Aydi, M. Abbas, and C. Vetro, "Partial Hausdorff metric and Nadler's fixed point theorem on partial metric spaces," Topology and Its Applications, vol. 159, no. 14, pp. 3234-3242, 2012.

[20] F. S. de Blasi, J. Myjak, S. Reich, and A. J. Zaslavski, "Generic existence and approximation of fixed points for nonexpansive set-valued maps," Set-Valued and Variational Analysis, vol. 17, no. 1, pp. 97-112, 2009. 
[21] L. Cirić, "Multi-valued nonlinear contraction mappings," Nonlinear Analysis: Theory, Methods \& Applications, vol. 71, no. 7-8, pp. 2716-2723, 2009.

[22] Y. Enjouji, M. Nakanishi, and T. Suzuki, "A generalization of Kannan's fixed point theorem," Fixed Point Theory and Applications, vol. 2009, Article ID 192872, 2009.

[23] Y. Feng and S. Liu, "Fixed point theorems for multi-valued contractive mappings and multi-valued Caristi type mappings," Journal of Mathematical Analysis and Applications, vol. 317, no. 1, pp. 103-112, 2006.

[24] M. Frigon, "Fixed point results for multivalued maps in metric spaces with generalized inwardness conditions," Fixed Point Theory and Applications, vol. 2010, Article ID 183217, pp. 1-19, 2010.

[25] S. Al-Homidan, Q. H. Ansari, and J.-C. Yao, "Some generalizations of Ekeland-type variational principle with applications to equilibrium problems and fixed point theory," Nonlinear Analysis: Theory, Methods \& Applications, vol. 69, no. 1, pp. 126-139, 2008.

[26] J. R. Jachymski, "Caristi's fixed point theorem and selections of set-valued contractions," Journal of Mathematical Analysis and Applications, vol. 227, no. 1, pp. 55-67, 1998.

[27] H. Kaneko, "Generalized contractive multivalued mappings and their fixed points," Mathematica Japonica, vol. 33, no. 1, pp. 5764, 1988.

[28] M. Kikkawa and T. Suzuki, "Some similarity between contractions and Kannan mappings," Fixed Point Theory and Applications, vol. 2008, Article ID 649749, 8 pages, 2008.

[29] W. A. Kirk, "Fixed points of asymptotic contractions," Journal of Mathematical Analysis and Applications, vol. 277, no. 2, pp. 645-650, 2003.

[30] W. A. Kirk and L. M. Saliga, "The Brézis-Browder order principle and extensions of Caristi's theorem," Nonlinear Analysis: Theory, Methods \& Applications, vol. 47, no. 4, pp. 2765-2778, 2001.

[31] A. Latif and S. A. Al-Mezel, "Fixed point results in quasimetric spaces," Fixed Point Theory and Applications, vol. 2011, Article ID 178306, 2011.

[32] S. Leader and S. L. Hoyle, "Contractive fixed points," Fundamenta Mathematicae, vol. 87, pp. 93-108, 1975.

[33] N. Mizoguchi and W. Takahashi, "Fixed point theorems for multivalued mappings on complete metric spaces," Journal of Mathematical Analysis and Applications, vol. 141, no. 1, pp. 177$188,1989$.

[34] M. Nakanishi and T. Suzuki, "An observation on Kannan mappings," Central European Journal of Mathematics, vol. 8, no. 1, pp. 170-178, 2010.

[35] S. Park, "Characterizations of metric completeness," Colloquium Mathematicum, vol. 49, no. 1, pp. 21-26, 1984.

[36] H. K. Pathak and N. Shahzad, "Fixed point results for set-valued contractions by altering distances in complete metric spaces," Nonlinear Analysis: Theory, Methods \& Applications, vol. 70, no. 7, pp. 2634-2641, 2009.

[37] Q. Kiran and T. Kamran, "Nadler's type principle with high order of convergence," Nonlinear Analysis: Theory, Methods \& Applications, vol. 69, no. 11, pp. 4106-4120, 2008.

[38] S. Reich, "Fixed points of contractive functions," Bollettino dell'Unione Matematica Italiana, vol. 4, pp. 26-42, 1972.

[39] S. Reich, "Some problems and results in fixed point theory," Contemporary Mathematics, vol. 21, pp. 179-187, 1983.
[40] S. Reich and A. J. Zaslavski, "Generic existence of fixed points for set-valued mappings," Set-Valued Analysis, vol. 10, no. 4, pp. 287-296, 2002.

[41] S. Reich and A. J. Zaslavski, Genericity in Nonlinear Analysis, vol. 34 of Developments in Mathematics, Springer, New York, NY, USA, 2014.

[42] N. Shioji, T. Suzuki, and W. Takahashi, "Contractive mappings, Kannan mappings and metric completeness," Proceedings of the American Mathematical Society, vol. 126, no. 10, pp. 3117-3124, 1998.

[43] W. Sintunavarat and P. Kumam, "Common fixed point theorem for cyclic generalized multi-valued contraction mappings," Applied Mathematics Letters, vol. 25, no. 11, pp. 1849-1855, 2012.

[44] T. Suzuki, "Several fixed point theorems concerning $\tau$-distance," Fixed Point Theory and Applications, no. 3, pp. 195-209, 2004.

[45] T. Suzuki, "Several fixed point theorems in complete metric spaces," Yokohama Mathematical Journal, vol. 44, no. 1, pp. 6172, 1997.

[46] T. Suzuki, "Mizoguchi-Takahashi's fixed point theorem is a real generalization of Nadler's," Journal of Mathematical Analysis and Applications, vol. 340, no. 1, pp. 752-755, 2008.

[47] T. Suzuki, "Subrahmanyam's fixed point theorem," Nonlinear Analysis: Theory, Methods \& Applications, vol. 71, no. 5-6, pp. 1678-1683, 2009.

[48] T. Suzuki, "Convergence of the sequence of successive approximations to a fixed point," Fixed Point Theory and Applications, vol. 2010, Article ID 716971, 14 pages, 2010.

[49] T. Suzuki, "Contractive mappings are Kannan mappings, and Kannan mappings are contractive mappings in some sense," Commentationes Mathematicae, vol. 45, no. 1, pp. 43-56, 2005.

[50] T. Suzuki and W. Takahashi, "Fixed point theorems and characterizations of metric completeness," Topological Methods in Nonlinear Analysis, vol. 8, no. 2, pp. 371-382, 1996.

[51] W. Takahashi, "Existence theorems generalizing fixed point theorems for multivalued mappings," in Fixed Point theory Theory and Applications (Marseille, 1989), J. B. Baillon and M. Théra, Eds., vol. 252 of Pitman Research Notes in Mathematics Series, pp. 397-406, Longman Sci. Tech., Harlow, UK, 1991.

[52] E. Tarafdar, "An approach to fixed-point theorems on uniform spaces," Transactions of the American Mathematical Society, vol. 191, pp. 209-225, 1974.

[53] E. Tarafdar and G. X.-Z. Yuan, "Set-valued topological contractions," Applied Mathematics Letters, vol. 8, no. 6, pp. 79-81, 1995.

[54] K. Włodarczyk, R. Plebaniak, and M. Doliński, "Cone uniform, cone locally convex and cone metric spaces, endpoints, setvalued dynamic systems and quasi-asymptotic contractions," Nonlinear Analysis: Theory, Methods \& Applications, vol. 71, no. 10, pp. 5022-5031, 2009.

[55] K. W. Włodarczyk and R. Plebaniak, "Periodic point, endpoint, and convergence theorems for dissipative set-valued dynamic systems with generalized pseudodistances in cone uniform and uniform spaces," Fixed Point Theory and Applications, vol. 2010, Article ID 864536, pp. 1-32, 2010.

[56] K. Włodarczyk, R. Plebaniak, and C. Obczyński, “Convergence theorems, best approximation and best proximity for set-valued dynamic systems of relatively quasi-asymptotic contractions in cone uniform spaces," Nonlinear Analysis: Theory, Methods \& Applications, vol. 72, no. 2, pp. 794-805, 2010.

[57] K. Włodarczyk and R. Plebaniak, "Quasigauge spaces with generalized quasipseudodistances and periodic points of dissipative set-valued dynamic systems," Fixed Point Theory and Applications, vol. 2011, Article ID 712706, pp. 1-23, 2011. 
[58] K. Włodarczyk and R. Plebaniak, "Kannan-type contractions and fixed pointsin uniform spaces," Fixed Point Theory and Applications, vol. 2011, p. 90, 2011.

[59] K. Włodarczyk and R. Plebaniak, "Contractivity of Leader type and fixed points in uniform spaces with generalized pseudodistances," Journal of Mathematical Analysis and Applications, vol. 387, no. 2, pp. 533-541, 2012.

[60] K. Włodarczyk and R. Plebaniak, "Generalized uniform spaces, uniformly locally contractive set-valued dynamic systems and fixed points," Fixed Point Theory and Applications, vol. 2012, article 104, pp. 1-39, 2012.

[61] K. Włodarczyk and R. Plebaniak, "Leader type contractions, periodic and fixed points and new completivity in quasi-gauge spaces with generalized quasi-pseudodistances," Topology and its Applications, vol. 159, no. 16, pp. 3504-3512, 2012.

[62] K. Włodarczyk and R. Plebaniak, "Fixed points and endpoints of contractive set-valued maps in cone uniform spaces with generalized pseudodistances," Fixed Point Theory and Applications, vol. 2012, article 176, 2012.

[63] K. Włodarczyk and R. Plebaniak, "Contractions of Banach, Tarafdar, Meir-Keeler, Ćirić-Jachymski-Matkowski and Suzuki types and fixed points in uniform spaces with generalized pseudodistances," Journal of Mathematical Analysis and Applications, vol. 404, no. 2, pp. 338-350, 2013.

[64] K. Włodarczyk and R. Plebaniak, "Asymmetric structures, discontinuous contractions and iterative approximation of fixed and periodic points," Fixed Point Theory and Applications, vol. 2013, article 128, 2013.

[65] K. Włodarczyk and R. Plebaniak, "New completeness and periodic points of discontinuous contractions of Banach-type in quasi-gauge spaces without Hausdorff property," Fixed Point Theory and Applications, vol. 2013, article 289, pp. 1-27, 2013.

[66] R. Plebaniak, "On best proximity points for set-valued contractions of Nadler type with respect to $b$-generalized pseudodistances in b-metric spaces," Fixed Point Theory and Applications, vol. 2014, article 39, 2014.

[67] C.-K. Zhong, J. Zhu, and P.-H. Zhao, "An extension of multivalued contraction mappings and fixed points," Proceedings of the American Mathematical Society, vol. 128, no. 8, pp. 24392444, 2000. 


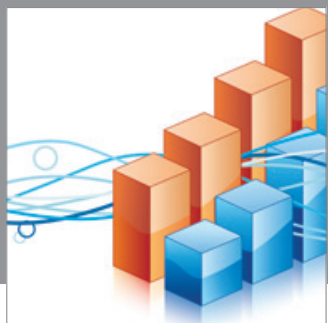

Advances in

Operations Research

mansans

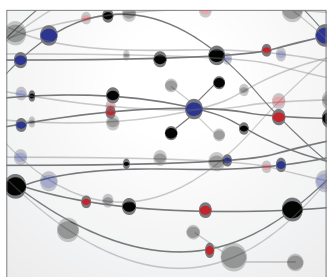

The Scientific World Journal
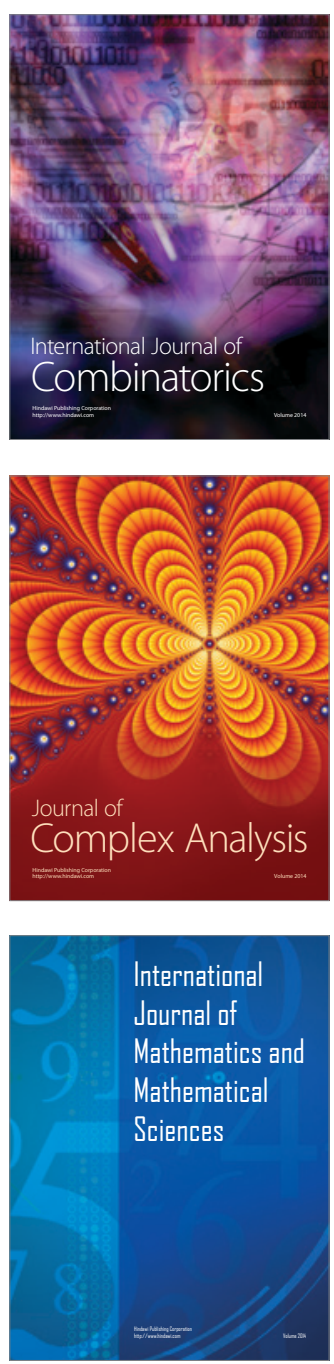
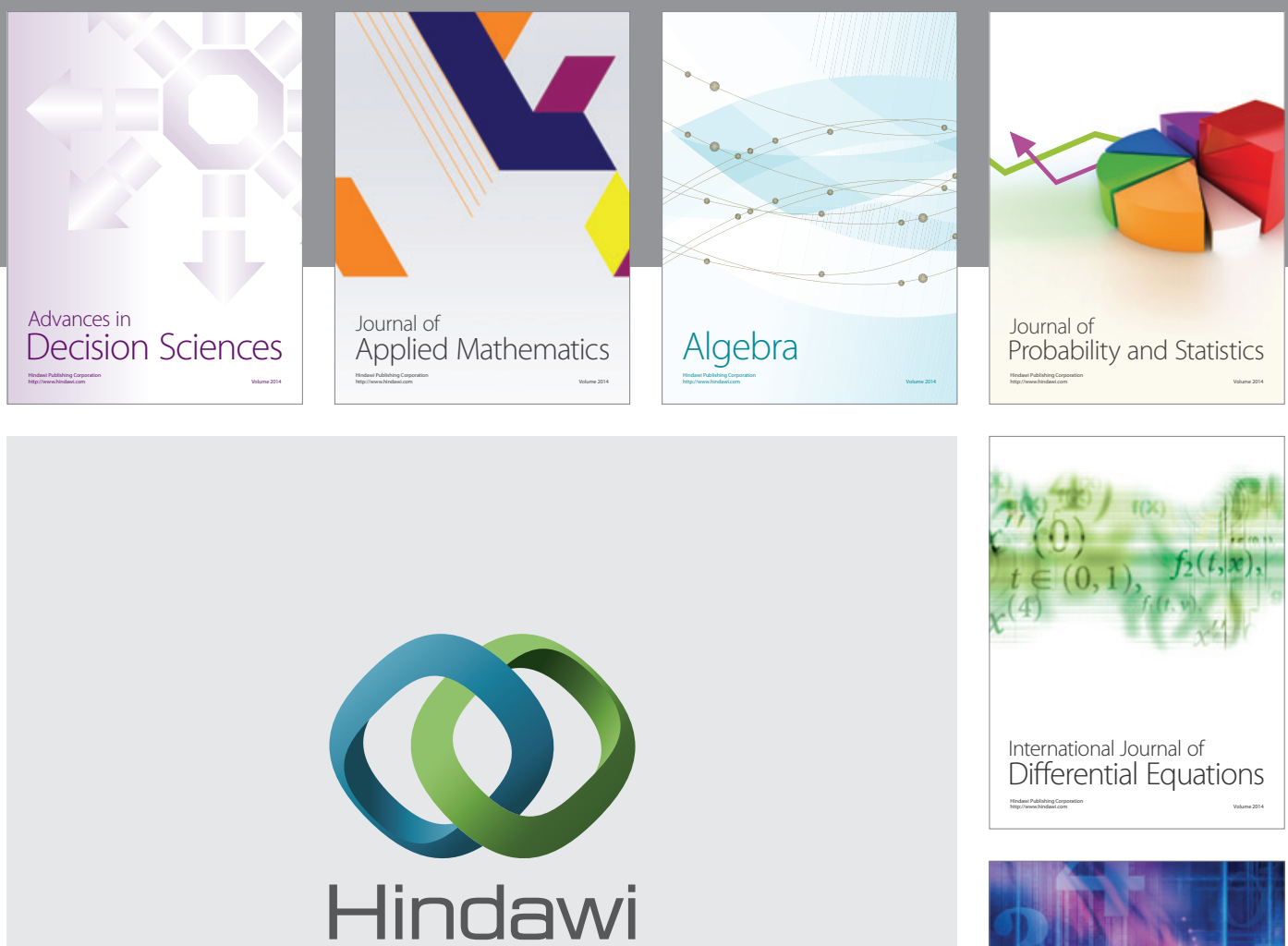

Submit your manuscripts at http://www.hindawi.com
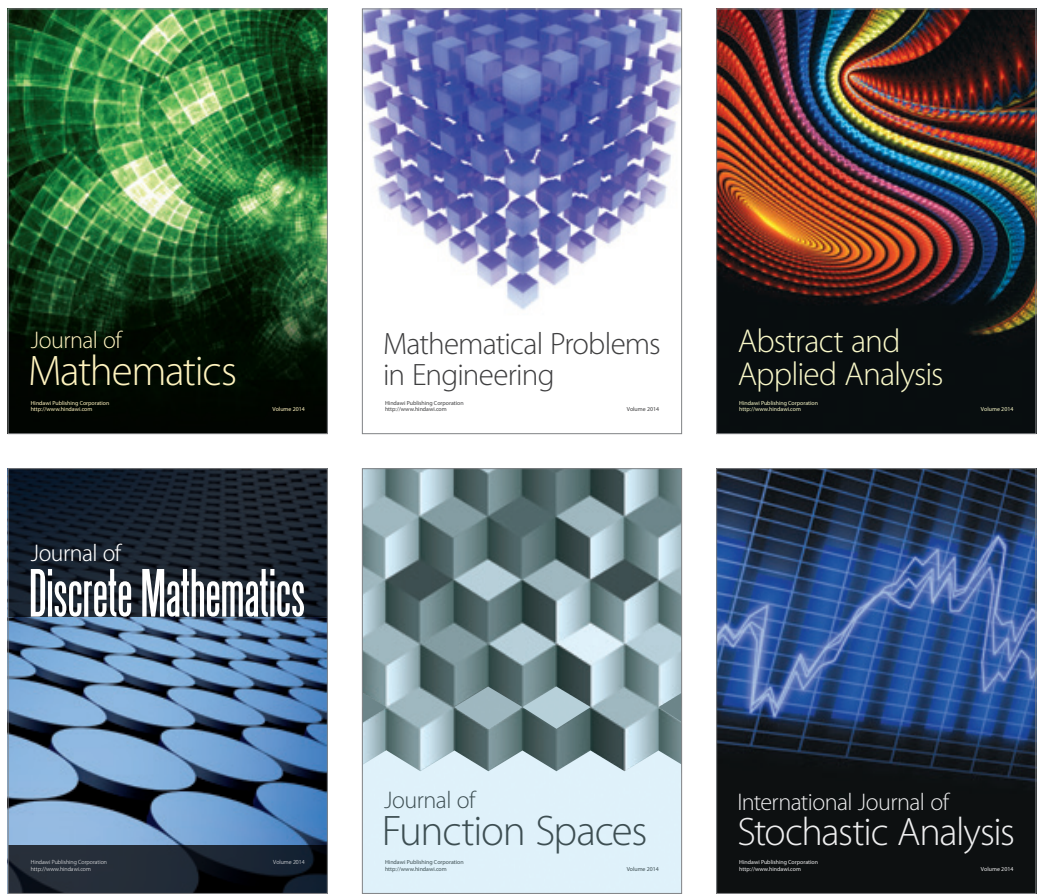

Journal of

Function Spaces

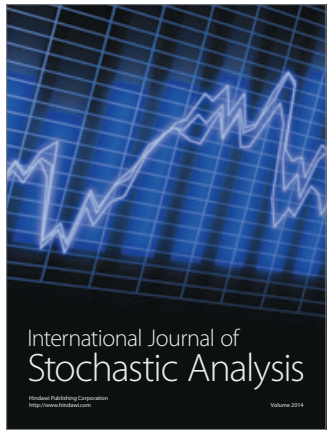

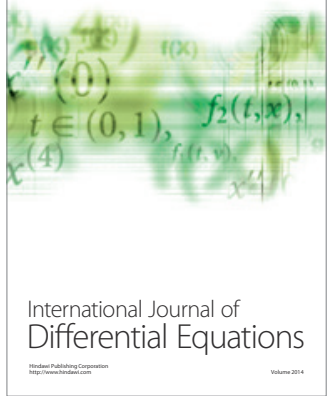
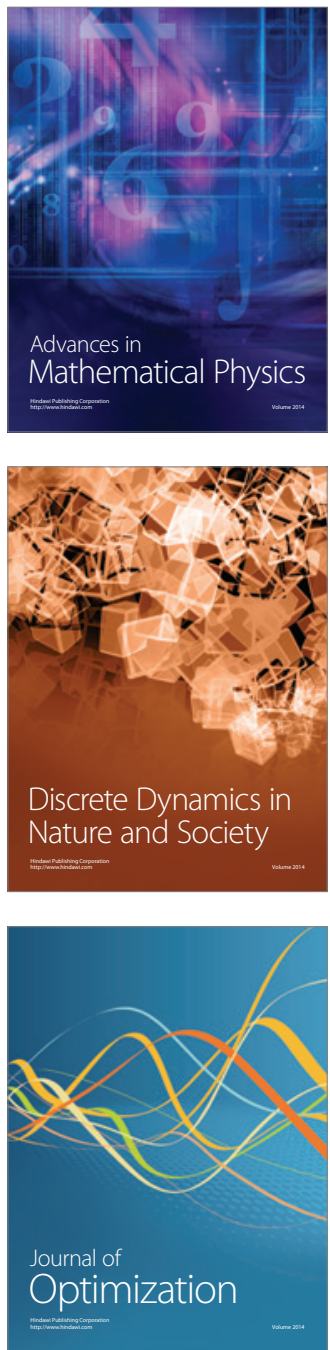